\title{
Molecular evolution of classic Hodgkin lymphoma revealed through whole genome sequencing of Hodgkin and Reed Sternberg cells
}

Francesco Maura ${ }^{1}$, Bachisio Ziccheddu ${ }^{1}$,Jenny Z. Xiang ${ }^{2,3}$, Bhavneet Bhinder ${ }^{3}$, Federico Abascal $^{4}$, Kylee H. Maclachlan ${ }^{5}$, Kenneth Wha Eng ${ }^{3}$, Manik Uppal ${ }^{3}$, Feng $\mathrm{He}^{2}$, Wei Zhang $^{2}$, Qi Gao ${ }^{6}$, Venkata Yellapantula ${ }^{5,7}$, Sunita Park ${ }^{8}$, Matthew Oberley ${ }^{9}$, Elizabeth Ruckdeschel $^{10}$, Megan S. Lim ${ }^{11}$, Gerald Wertheim ${ }^{11}$, Matthew Barth ${ }^{12}$, Terzah M. Horton $^{13}$, Christopher Forlenza ${ }^{6}$, Yanming Zhang ${ }^{6}$, Ola Landgren ${ }^{1}$, Craig H Moskowitz ${ }^{1}$, Ethel Cesarman ${ }^{2}$, Marcin Imielinski ${ }^{2,3,5}$, Olivier Elemento ${ }^{2,3}$, Mikhail Roshal ${ }^{6}$, Lisa Giulino-Roth ${ }^{2}$

${ }^{1}$ Sylvester Comprehensive Cancer Center, University of Miami, Miami, FL, USA.

${ }^{2}$ Weill Cornell Medical College, New York, NY USA

${ }^{3}$ Englander Institute for Precision Medicine, Institute for Computational Biomedicine, and Meyer Cancer Center, Weill Cornell Medical College, New York, NY, USA.

${ }^{4}$ The Cancer, Ageing and Somatic Mutation Programme, Wellcome Sanger Institute, Hinxton, Cambridgeshire, CB10 1SA, UK.

${ }^{5}$ Myeloma Service, Department of Medicine, Memorial Sloan Kettering Cancer Center, New York, NY, USA.

${ }^{6}$ Memorial Sloan Kettering Cancer Center, New York, NY, USA

${ }^{7}$ Department of Pathology and Laboratory Medicine at Children's Hospital Los Angeles, CA, USA.

${ }^{8}$ Department of Pathology, Children's Hospital of Atlanta, Atlanta, GA, USA.

${ }^{9}$ Caris Life Sciences 4610 South 44th Place Phoenix, AZ 84050;

${ }^{10}$ Department of Pathology, Upstate Medical University, Syracuse, NY USA.

${ }^{11}$ Department of Pathology, Children's Hospital of Philadelphia, Philadelphia, PA USA.

${ }^{12}$ Department of Pediatrics, Roswell Park Comprehensive Cancer Center, Buffalo, NY USA.

${ }^{13}$ Department of Pediatrics, Baylor College of Medicine, Houston, TX USA.

Running Title: Whole-genome sequencing of Hodgkin lymphoma

Key words: classic Hodgkin Lymphoma, Reed-sternberg cell, whole genome sequencing, germinal center, mutational signatures, timing

\section{Corresponding Authors:}

Francesco Maura, MD,

Sylvester Comprehensive Cancer Center, University of Miami, 1120 NW 14th Ave, Clinical Research Building

Miami, FL 33136

Phone (305) 2437687

E-mail: fxm557@med.miami.edu 
45 Mikhail Roshal

461275 York Avenue,

47 New York, NY, 10065, Suite C563

$48 \quad$ T 212.639.6091

49 Email: roshalm@mskcc.org

50

51 Lisa Giulino-Roth

52525 East $68^{\text {th }}$ Street

53 Payson 695

54 New York, NY 10065

55 Phone: (212) 746-3494

56 Email: lgr2002@med.cornell.edu

57

58 


\section{Abstract}

60 The rarity of malignant Hodgkin and Reed Sternberg (HRS) cells within a classic

61 Hodgkin lymphoma (cHL) biopsy limits the ability to study the genomics of $\mathrm{cHL}$. To

62 circumvent this, our group has previously optimized fluorescence-activated cell sorting

63 to purify HRS cells. Here we leveraged this method to report the first whole genome

64 sequencing landscape of HRS cells and reconstruct the chronology and likely etiology

65 of pathogenic events prior to the clinical diagnosis of $\mathrm{cHL}$. We identified alterations in

66 driver genes not previously described in $\mathrm{CHL}$, a high activity of the APOBEC mutational

67 signature, and the presence complex structural variants including chromothripsis. We

68 found that the high ploidy observed in $\mathrm{cHL}$ is often acquired through multiple,

69 independent large chromosomal gain events including whole genome duplication. The

70 first of these likely occurs several years prior to the diagnosis of $\mathrm{cHL}$, and the last gains

71 typically occur very close to the time of diagnosis. Evolutionary timing analyses revealed

72 that driver mutations in B2M, BCL7A, GNA13, and PTPN1, and the onset of AID driven

73 mutagenesis usually preceded large chromosomal gains. The study provides the first

74 temporal reconstruction of $\mathrm{cHL}$ pathogenesis and suggests a relatively long time course

75 between the first pathogenic event and the clinical diagnosis. 


\section{Article}

Classic Hodgkin lymphoma ( $\mathrm{cHL})$ is characterized by a unique pathological composition where a small fraction of Hodgkin and Reed Sternberg (HRS) tumor cells

$80(\sim 1 \%)$ are surrounded by an extensive and complex immune and stromal infiltrate. ${ }^{1}$ The

81 paucity of HRS cells in tumor tissue has precluded the genomic investigation of $\mathrm{CHL}$

82 using standard platforms. Our group has optimized fluorescence-activated cell sorting

83 (FACS) to isolate HRS cells and intratumor B- and T- cells and perform whole exome

84 sequencing (WES). ${ }^{2}$ Data on HRS exomes from our group and others have revealed

85 mutations in critical driver genes involved in NF-кB and JAK/STAT signaling pathways

86 as well as genes involved in immune escape. ${ }^{2-5}$ These investigations, however, were

87 limited to captured coding sequences, and therefore not able to comprehensively

88 decipher the genomic complexity of cHL. Furthermore, the chronologic order in which

89 somatic alterations are acquired is largely unknown in $\mathrm{cHL}$, limiting our understanding of

90 the earliest drivers/initiating events.

91 Whole genome sequencing (WGS) has the potential to fully characterize the

92 somatic genomic landscape including: i) the catalogue of coding and non-coding

93 mutations, ii) large and focal copy number alterations (CNA), iii) structural variants (SV)

94 including complex events, and iv) mutational processes involved in cancer

95 pathogenesis. ${ }^{6}$ Here we performed WGS on FACS-isolated HRS cells and matched

96 normal tissue from 25 patients with $\mathrm{cHL}$ and WES from an additional 36 cases. We

97 combined CNA, single nucleotide variant (SNV) and SV analyses to decipher the

98 landscape and chronological order of key driver events in $\mathrm{cHL}$. Altogether these

99 analyses reveal previously unknown insights into the pathogenesis of $\mathrm{cHL}$ and pave the 
100 way for the design of novel therapeutic strategies targeting early driver events in a

101 disease that frequently affects children and young adults.

102

\section{Results}

104 Single nucleotide variants in classical Hodgkin lymphoma driver genes.

105 To evaluate the genome of HRS cells, HRS and intra-tumoral T-cells were

106 isolated from cHL biopsies using FACS as previously described. ${ }^{7}$ Intra-tumoral T-cells

107 from each case were used as the germline control. We interrogated WGS from 25

108 cases of $\mathrm{cHL}$ including 10 pediatric cases (age<18), 9 cases in adolescents and young

109 adults (AYA, age 18-40), and 6 in older adults (age>40) (Supplemental Table 1).

110 Twenty-two (88\%) of the cases were obtained at the time of diagnosis and $3(12 \%)$

111 were obtained at the time of relapse. The median DNA input from HRS cells was 13.6ng

112 (range 4.2 - 226ng). Given the ultra-low input, sequencing data were generated by

113 amplifying the DNA (median 10 amplification cycles, range 7-15). To ensure a robust

114 quality control, we explored possible amplification-based mutational artifacts across the

115 genome, observing an enrichment of single base substitution (SBS) within distinct

116 trinucleotide context reflecting palindromic artifacts (Supplemental Figure $\mathbf{1}$ and

117 Supplemental Table 2). ${ }^{8,9}$ After having removed these amplification-induced

118 palindromic sequencing artifacts, we observed a median mutational burden of 5006 per

119 genome (range 1763-18436), which is comparable to other aggressive lymphomas

120 (Figure 1a). ${ }^{10}$ We found that pediatric and AYA patients age $\leq 40 \mathrm{y}$ had a significantly

121 higher mutational burden than older adults over age 40 (median 5279 vs. 2945,

$122 p=0.009$, Figure 1b). This effect was not related to the sequencing coverage $(p=0.1)$. 
To increase the sample size for discovery analysis of driver mutations, we

124 performed WES on HRS cells from additional $36 \mathrm{cHL}$ cases, 10 of which have been

125 previously reported ${ }^{2}$ (Supplemental Table 3). After having identified and removed the

126 same amplification-induced sequencing palindromic artifact, we combined the two

127 cohorts [WGS ( $n=25)$ WES $(n=36)$ ] to perform a driver mutation discovery analysis. To

128 identify genes that are hit by nonsynonymous mutations more frequently than what

129 would be expected by chance (i.e. genes under positive selection), we ran $d n d s v^{11}$ both

130 on all genes and, to increase statistical power, on a restricted set of cancer genes built

131 by combining the COSMIC census catalogue with genes previously reported as

132 recurrently mutated in $\mathrm{cHL}(\mathrm{n}=15) .{ }^{2-5}$ Overall, dndscv detected 23 genes under positive

133 selection $(\mathrm{q}<0.1)$ and five hotspots (CCND3, TP53, B2M, EZH2, and XPO1) (Figure 1c,

134 Supplemental Table 4-5). Ninety-five percent of $\mathrm{cHL}$ cases had at least one of these

135 driver genes mutated, with an average of 3.38 (range 2.45-3.98) drivers per sample.

136 Pediatric/AYA and older adult cases did not differ in the estimated number of mutations

137 in driver genes calculated based on the global ratio of nonsynonymous to synonymous

138 mutations.

139 The most common driver alterations were in SOCS1 (62\% of cases), TNFAIP3

$140(36 \%)$ and B2M (32\%). We observed alterations in 11 driver genes not previously

141 described in $\mathrm{CHL}$ (Supplemental Table 4), ${ }^{2-5,12}$ including BCL7A and CISH. BCL7A

142 encodes a subunit of the SWI/SNF complex and has been described as a tumor

143 suppressor in B-cell non-Hodgkin lymphomas. ${ }^{13,14}$ Cytokine inducible SH2 containing

144 protein $(\mathrm{CISH})$ is a member of the SOCS family which regulates cytokine receptor

145 signaling through the JAK/STAT pathway. ${ }^{15}$ 
$B 2 M, B C L 7 A, G N A 13, I T P K B$, and SOCS1 tended to have more than one

147 nonsynonymous mutation in the same patient (Figure 1c). Using the WGS mutational

148 distribution on the entire footprint of these hypermutated genes, we observed that

$149 B C L 7 A$, and ITPKB showed a higher non-coding:coding mutation ratio compared to

150 other driver genes (FDR $<0.1$ in Fisher's exact test). Most of these mutations were

151 compatible with the AID mutational signature (AID/SBS84 COSMIC signature;

152 Supplemental Figure 2). ${ }^{10,16-18}$ The SOCS1 mutational profile was also driven by

153 AID/SBS84 mutational activity however the non-coding:coding mutation ratio was not

154 significantly higher compared to other drivers. The presence of localized AID

155 hypermutation activity, likely related to somatic hypermutation (SHM), provides a

156 mechanistic explanation of why these genes tend to be mutated multiple times in the

157 same patient. In contrast, B2M and GNA13 were recurrently mutated more than once

158 without enrichment for either intronic or SHM/AID mutations (Supplemental Figure 2).

160 Copy number alterations (CNA)

161 Combining WGS and WES data, we investigated the somatic CNA landscape in

$162 \mathrm{cHL}$. Consistent with the frequent multinucleated nature of HRS cells, we observed high

163 ploidy (median 2.95, range 1.66-5.33). Despite the well characterized bi- and multi-

164 nucleated phenotype of Reed Sternberg cells, not all patients had whole genome

165 duplication (WGD, 54\%). This was validated by FISH in six patients with available

166 material (Supplemental Figure 3). This suggests that multinucleation is not universally

167 associated with WGD in cHL. ${ }^{19-21}$ 
Running GISTIC2.0, ${ }^{22}$ which identifies genes and chromosomal segments

169 recurrently targeted by somatic CNA, we identified 19 recurrent CNA peaks: 5 gains and

17014 losses (Figure 2a, and Supplemental Table 6-7). The majority of these CNAs were

171 caused by either large (>10 Mb) or whole chromosome/arm events (95.6\%;

172 Supplemental Figure 4-5). Matching GISTIC CNA peaks with genes that are either

173 positively selected in $\mathrm{cHL}$ and/or reported in the COSMIC census, we identified 4 genes

174 recurrently involved in amplifications and 17 by deletions. For example, in TNFAIP3, the

175 most frequently deleted gene (44\% of all patients), $77 \%$ of deletions were either large

176 (>10 Mb) or whole arm/chromosome loss. The most frequently amplified loci were

177 9p24.1 (PDCD1LG2/JAK2/CD274), and 2p16.1 (REL/XPO1), (67\% and 85\% of patients

178 respectively, Supplemental Figure 4). These amplifications were similarly mostly

179 caused by large and whole chromosome/arm duplications ( $80 \%$ and $85 \%$ respectively).

180 Overall, our data suggests that known $\mathrm{cHL}$ driver genes are recurrently involved in large

181 CNA, and, in a smaller proportion, by focal events. We also observed a high prevalence

182 of high-level gains (>6 copies) in 2p16.1 - REL/XPO1 [median 11 copies (range 7-31);

$183 \mathrm{n}=10,16 \%$ ] and 9p24.1 - PDCD1LG2/JAK2/CD274 [median 8 copies, range 6.3-13);

$184 \mathrm{n}=11,18 \%]$ (Figure 2b).

185 Next, we combined CNA data and nonsynonymous mutations to investigate bi-

186 allelic events involving driver genes extracted by GISTIC2.0 $(\mathrm{n}=21)$ and by $d n d s c v$

187 ( $\mathrm{n}=25)$. TNFAIP3 $(\mathrm{n}=15 ; 24 \%), B 2 M(\mathrm{n}=10 ; 16 \%)$, GNA13 $(\mathrm{n}=9 ; 15 \%)$ were the most

188 common driver genes with bi-allelic inactivation. This was mostly driven by deletion on

189 one allele and a nonsynonymous mutation on the other (Figure 2b). 


\section{Timing of $\mathrm{CHL}$ driver alterations}

192 Next, we investigated the relative timing of driver mutation acquisition with

193 respect to chromosomal gains. For this analysis we included chromosomal gains of any

194 size and level. By leveraging the high $\mathrm{cHL}$ ploidy and high prevalence of chromosomal

195 gains, we performed a comprehensive investigation of the relative timing of driver

196 mutation acquisition with respect to the chromosomal gain in 61 patients (Figure 2c). ${ }^{23-}$

$197{ }^{26}$ Clonal mutations within chromosomal gains can have one of two purity-corrected

198 variant allelic frequencies (VAFs): i) $\leq 33 \%$ reflecting mutations acquired either on one of

199 the two duplicated alleles after the gain or on the minor allele non-duplicated allele; ii)

$200 \geq 66 \%$ reflecting mutations acquired on the duplicated allele before the duplication. A

201 large fraction of mutations in driver genes $(140 / 227 ; 61 \%)$ showed a duplicated VAF

$202(\geq 66 \%)$, suggesting that they were acquired prior to chromosomal gains (Figure 2c).

203 Loss of function mutations involved by copy neutral loss of heterozygosity (LOH)

204 showed the same pattern, with the mutation often proceeding the chromosomal

205 duplication. Next, we estimated the chronological order of mutations in $25 \mathrm{cHL}$ driver

206 genes/hot spots (Figure 2d). Temporal estimates were generated by the Bradley Terry

207 model based on the integration between the CCF and duplication status of all mutations

208 involving the 25 driver genes. Mutations in PTPN1, GNA13, XPO1, HIST1H1E and B2M

209 emerged as early drivers occurring prior to other mutations and prior to chromosomal

210 gain events.

211

212 Mutational signatures 
213 To investigate which mutational processes are involved in $\mathrm{cHL}$, we used both 214 sigProfiler and the hierarchical Dirichlet process (hdp) identifying 5 main mutational

215 signatures [or single base substitution (SBS) signatures], and subsequently mmsig to

216 accurately estimate their activity and contribution (Figure 3a-b)..$^{10,17,26-29}$ SBS1 and

217 SBS5, the so-called clock-like mutational processes, were detected in all patients

218 (median of 4250 mutations, range $735-11159$ ). ${ }^{30}$ Seventy-two percent of patients had

219 evidence of APOBEC mutational activity (SBS2 and SBS13), (Figure 3b-c). This high

220 prevalence of APOBEC is similar to what has been reported based on HL WES data ${ }^{5}$

221 and confirms the strong APOBEC pathogenetic role in cHL. Four patients (16\%)

222 showed a particularly high APOBEC contribution mostly driven by a major APOBEC3A

223 activity compared to APOBEC3B (hyper APOBEC), in line with what has been reported

224 in other cancers. ${ }^{26,31}$ The other $14(84 \%)$ were characterized an APOBEC3A:3B ratio $\sim 1$

225 ("canonical" APOBEC). Looking at the APOBEC contribution before and after large 226 chromosomal gains, we observed an enrichment among the non-duplicated mutations

227 compared to the duplicated reflecting a later role in cancer pathogenesis (i.e., after the

228 gain; $p=0.002$ using paired Wilcoxon test; Figure $3 \mathrm{~d}$ ). ${ }^{32}$ In contrast to other cancers like

229 multiple myeloma, ${ }^{23,26}$ APOBEC activity was also detectable before the chromosomal

230 gains, suggesting that large chromosomal duplications often occur in a clone in which

231 APOBEC is already active.

232 One out of three patients whose sample was collected at relapse (IID_H198450)

233 showed a clear presence of SBS25, a mutational signature previously linked to a still

234 unknown chemotherapy agent. This signature has been observed in normal colorectal

235 crypts in a patient previously treated with chemotherapy for non-Hodgkin lymphoma ${ }^{33}$ 
236 and in $\mathrm{cHL}_{\text {cell lines }}{ }^{10}$. This is the first report of SBS25 in relapsed $\mathrm{cHL}$, demonstrating

237 that similar to other cancers, $\mathrm{cHL}$ can acquire hundreds of mutations after exposure to

238 distinct chemotherapy agents. ${ }^{10,26,34-36}$ The presence of a large SBS25 clonal mutational

239 burden can be seen as a unique barcoding, reflecting the expansion of one single tumor

240 cell that survived front-line treatment and subsequently took the clonal dominance. ${ }^{35-37}$

241 Leveraging this concept and estimating the SBS25 contribution before and after the

242 three large chromosomal gains in this case, we observed chemotherapy-related

243 mutations both before and after the large chromosomal gains. The presence of

244 chemotherapy related mutations preceding large chromosomal gains indicates that

245 these CNA events were acquired after exposure to chemotherapy.

Molecular timing of multi-chromosomal gain events

249 (e.g., WGD). Although most of these events are clonal, they may not have been

250 acquired at the same time. To explore the temporal pattern of acquisition of

251 chromosomal gains, including WGD, in $\mathrm{cHL}$, we evaluated the corrected proportion of

252 duplicated and non-duplicated clonal mutations within large chromosomal gains (i.e.,

253 molecular time approach). ${ }^{23}$ In line with our previous work, ${ }^{24}$ we restricted our analysis

254 to clonal chromosomal trisomy, tetrasomy, gains, and copy neutral LOH larger than 1

$255 \mathrm{Mb}$, with more than 50 clonal SNVs after having removed immunoglobulin loci and all

256 localized hypermutated events (i.e., kataegis). Overall, in $62 \%$ gains the molecular time

257 was higher than 0.5 (median 0.58; range $0.12-1.00$ ), reflecting an intermediate-late

258 acquisition pattern (Figure $4 \mathbf{a}-\mathbf{b}$ ). ${ }^{23}$ There were distinct events acquired particularly late, 
259 such as large gains on chromosome $2-R E L / X P O 1,6 q$ and $4 q$ (Figure 4c). Other 260 recurrent CNAs such as 9p24.1 - PDCD1LG2/JAK2/CD274 were often intermediate. In

$26112 / 20$ patients (60\%) the final clonal copy number profile was acquired through at least

262 two independent events (i.e., two groups of large gains with different molecular time,

263 Methods; Figure 4a-c and Supplemental Figure 6). This suggests that HRS cells

264 have a predisposition over time to acquire additional chromosomal gains.

265 To convert these relative estimations into absolute time (i.e., the age at which

266 these events were acquired in each patient's life), we utilized the clock-like mutational

267 signatures as previously described. ${ }^{10,25,26,30}$ SBS1 and SBS5 (Figure 3a-b) have been

268 reported to be universally present in all cells and to act in a constant rate over

269 time. $^{10,23,30}$ Based on this, we can quantify their activity before and after large

270 chromosomal gains and convert the clock-like-based molecular time to an absolute

271 value (i.e., years of life). We first confirmed that the SBS1 and SBS5 mutation rate were

272 constant over time (Figure $4 \mathbf{d}$ ). We observed a constant rate in pediatric and AYA

273 patients as well as in older adults. Of note, the rates differed among age groups with a

274 higher mutation rate in pediatric and AYA patients $(p=0.01)$. Estimating the SBS1- and

275 SBS5-based molecular time for large chromosomal gains acquired within the same time

276 window and converting these relative estimates into absolute ones, we observed that

277 the first multi-chromosomal gain event in $\mathrm{cHL}$ is often acquired several years before the

278 diagnosis/sample collection (median lag between chromosomal gain and diagnosis: 5.3

279 years, range 1.8-27.4) (Figure 4e). In cases where we could assess the timing of the

280 latest multi chromosomal gain events, it usually overlapped with the age at 
281 diagnosis/sample collection suggesting a potential role in the final lymphoma selection

282 and clonal expansion.

284 Structural variants and complex events

The high resolution of WGS allows us to perform the first characterization of the landscape of structural variants (SVs) in $\mathrm{cHL} .{ }^{38-40}$ Applying JaBbA we were able to infer

287 SV and CNA junction-balanced genome graphs with high fidelity allowing a detailed 288 characterization of both single and complex events. ${ }^{38}$ Overall, we observed clear 289 evidence of complex events such as chromothripsis $(n=4)$, double minutes $(d m, n=2)$, 290 breakage-fusion-bridge $(b f b ; n=4)$, pyrgo $(n=2)$, chromoplexy $(n=1)$ and templated

291 insertions $(\mathrm{n}=10)$ (Figure 5a). The role of single and complex events in the acquisition

292 of CNAs involving distinct drivers emerged as heterogenous. For example, while most

293 of the PDCD1LG2/JAK2/CD274 high level gains were caused by large whole arm 294 chromosomal gains or single events, in one patient these genes had multiple copies as 295 consequence of $d m$ (IID_H198450; Figure 5b). Similarly, in IID_H198427 a bfb event 296 was responsible for the acquisition of 18 copies of XPO1/REL (Figure 5c).

297 To estimate the timing of loss-of-function events and the acquisition of distinct 298 SVs, we utilized two approaches: ${ }^{24}$ 1) we linked SV breakpoints to the molecular time of 299 chromosomal gains caused by the same SV (Figure 5d-e); 2) we estimated the relative 300 time of SVs that occurred within large chromosomal gains based on the copy number of 301 the SV breakpoint (Figure 5f-g, Supplemental Figure 7; see Methods). Applying 302 these approaches, we were able to time the acquisition of single and complex events 303 observing two relevant SV/CNA temporal patterns. In the first, chromothripsis emerged 
304 as an early event in $\mathrm{cHL}$ pathogenesis proceeding WGD in 3/3 patients in which this

305 analysis was possible. (Figure $\mathbf{5 d - g}$ ). In the second temporal pattern, we observed

306 early acquisition of PTPN1 deletion (i.e., before chromosomal gains/WGD) in 4/5

307 patients, suggesting the early driver role of this gene in $\mathrm{cHL}$ pathogenesis

308 (Supplemental Figure 7a and 8). Combining SV and SNVs in driver genes, PTPN1

309 was involved in $36 \%(9 / 25)$ of $\mathrm{cHL}$, either as early deletion $(n=4)$ or $\mathrm{SBS}(n=5)$, each

310 duplicated by a subsequent chromosomal gain.

312 Using mutational signatures to identify the HRS cell-of-origin

313 The HRS cell-of-origin is suspected to be a B-cell that is unable to fully mature

314 due to an unproductive B-cell receptor (BCR)..$^{5,12,41-44}$ This model has been supported

315 by the detection of AID-mediated somatic hypermutation (SHM) on an unproductive

316 VDJ, and nonsynonymous mutations involving AID off-target genes. To molecularly

317 evaluate this model and the relationship between the GC and HRS cells, we explored

318 the mutational signature within the immunoglobulin loci (lg) and within the footprint of

319 genes known to be involved by AID off-target activity (e.g., PAX5, BCL6, XPO1). These

320 regions showed clear activity of AID/SBS84 (Figure 3a and Figure 6a-c). The presence

321 of AID/SBS84 on Ig and on AID off-target genes has been reported in GC-derived

322 malignancies. ${ }^{10,26,45-47}$ In these tumors, AID/SBS84 always co-occurs with a genome

323 wide mutational process historically called "non-canonical" AID (SBS9) and thought to

324 result from SHM. Contrary to this model, we did not observe any evidence of genome-

325 wide SBS9 activity in AID/SBS84+ HRS cells (Figure 6d) ${ }^{10,16,24,26,47-49}$. This 
326 demonstrates for the first time that SHM is independent and temporally unrelated to

327 SBS9.

328 Next, to reconstruct the V(D)J of HRS cells, we ran $\operatorname{lgCaller}^{50}$ and confirmed that

329 the VDJ rearrangements were unproductive in all but 3 patients (Figure 6e,

330 Supplemental Table 8). To investigate when these unproductive BCRs were affected

331 by SHM/AID, we focused on $\mathrm{Ig}$ and AID off-targets genes involved by large

332 chromosomal gains. We observed both duplicated and non-duplicated AID mutations

333 (Figure 6f) suggesting that, in the pre-cHL clone interaction with the GC precedes

334 chromosomal duplications and, in some patients, might have been prolonged over time.

335 Altogether this data molecularly confirms the $\mathrm{cHL}$ pathogenetic model in which a naive

336 B-cell enters the GC, is exposed to AID and SHM, but as a consequence of an

337 unproductive BCR fails to expand in the GC. The acquisition of distinct genomic drivers

338 acquired before and during the GC reaction likely allows the pre-HL clone to escape

339 negative selection in the GC (Figure 7). After being established and immortalized, in

340 order to progress into $\mathrm{cHL}$, the pre-cHL clone acquires additional driver events over

341 time, in particular complex genomic events such as aneuploidies, WGD and SVs.

\section{Discussion}

$344 \quad$ Human cancers are suspected to arise from a pre-malignant clone that evolves

345 over time and often can be detected years before diagnosis, in some cases even in the

346 peri-natal period. ${ }^{23,25}$ Understanding the chronology of mutational processes leading to

347 malignancy can help guide novel diagnostic strategies and treatment approaches

348 directed to early driver events. In this study, for the first time, we leveraged WGS 
349 resolution to elucidate a pathogenetic model for $\mathrm{cHL}$ whereby tumor development is

350 shaped by the acquisition and selection of multiple drivers across different time windows

351 over several years (Figure 7). In this model driver mutations in B2M, BCL7A, PTPN1

352 and GNA13 emerged as early events as did deletions in PTPN1 and complex SV

353 events such as chromothripsis. These early events largely occur before large

354 chromosomal duplications which are often acquired at in intermediate/late timepoint

355 which was determined to be still several years prior to diagnosis. This temporal pattern

356 is different from what is observed in other hematological malignancies such as acute

357 lymphoblastic leukemia, multiple myeloma and chronic lymphocytic leukemia, where

358 large chromosomal duplications tend to be early events, potential playing a cancer-

359 initiating role. ${ }^{17,23,25,26,30,51}$

360 We also observed several key differences in the mutational landscape of HRS

361 cells across age groups. When compared to older adults age $>40 \mathrm{y}$, pediatric and AYA

362 patients were found to have a higher mutational burden genome-wide, and an

363 accelerated mutation rate in the clock-like signatures SBS1 and SBS5. This age

364 segregation correlates with the two epidemiologic peaks of $\mathrm{cHL}$ which occur in AYAs

365 age 15-40y and older adults age>55y, and suggests that the biology and the conditions

366 in which $\mathrm{cHL}$ develop across these two peaks might be distinct. Additional studies are

367 needed to investigate if the characteristics observed in younger patients with $\mathrm{cHL}$ may

368 be due to accelerated B-cell aging.

369 The HRS cell mutational signature landscape revealed the first robust

370 confirmation that the SBS9 signature represents a distinct GC mutational process

371 independent from AID and SHM. The original term "non-canonical-AID" was first 
372 reported because, across the lymphoproliferative disorders tested (follicular lymphoma,

373 CLL, multiple myeloma, Burkitt and diffuse large B-cell lymphomas), SBS9 always co-

374 occurred with AID/SBS84 and SHM. ${ }^{10,16,17,26,46,47}$ This model has been recently

375 challenged by the observations that: 1) AID/SBS84 can be active in CSR loci in CLL

376 with unmutated immunoglobulin heavy-chain variable region gene in the absence of

377 SBS9, 2) analysis of SBS9 genomic distribution in both tumor and normal B-cell

378 genomes, and 3) by the fact that SBS9 profile seems to reflect polymerase-eta repair

379 activity. ${ }^{16,17,52,53}$ In this study, the presence of all the GC hallmarks of SHM and AID

380 activity with the absence of SBS9 in $\mathrm{cHL}$ fills an important gap in our understanding of

381 which mutational processes are involved in both normal and pathological activity within

382 the GC.

383 Lastly, with WGS comprehensive resolution, we confirmed and expanded the

$384 \mathrm{cHL}$ pathogenic model proposed 30 years ago by Kupfer and collegues. ${ }^{41-44}$

385 Specifically, the interaction between pre-malignant cells and the GC emerged as a

386 critical phase for $\mathrm{cHL}$ development. During this likely prolonged encounter, the pre-

387 malignant cell survives and escapes the negative selection despite its unproductive

388 BCR, potentially due to early and concomitant acquisition of genomic drivers. Overall,

389 this study provides a critical new perspective on $\mathrm{cHL}$ pathogenesis and sheds light into

390 which mutational processes are involved in the interaction between the GC and B-cells.

\section{Methods}

393 Sorting: 
The HRS, $B$ and $T$ cell sorting was performed essentially as describe

395 previously. ${ }^{2,7}$ Briefly, single cell suspensions from CHL tumors containing up to $1 \times 10^{8}$

396 cells were either taken fresh or rapidly defrosted at $37^{\circ} \mathrm{C}$, washed in $50 \mathrm{~mL}$ of RPMI

$3971640 / 20 \%$ fetal bovine serum solution. The cells were stained with an antibody cocktail

398 composed of CD64 FITC (22; Beckman Coulter (BC), Brea, CA), CD30-PE (HRS4; BC)

399 CD5-BV510 (L17F12; Beckton-Dickinson (BD), San Jose, CA); CD40-PerCP-eFluor

400710 (5C3; eBiosciences, San Diego, CA); CD20-PC7 (B9E9; BC); CD15-APC (HI98;

401 BD); CD71 APC-A700 (YDJ1.2.2, BC); CD45 APC-H7 (2D1; BD), and CD95-Pacific

402 Blue (DX2; Life Technologies, Grand Island, NY). All sorting experiments were

403 performed on an FACS Aria-Fusion special-order research sorter using a 130- $\mu \mathrm{m}$

404 nozzle at 12 psi, acquiring up to $5 \times 10^{7}$ cells and collecting HRS, B, and T cells from

405 the tumor using a 3-way sort.

406

407 Whole genome sequencing

408 Sample library construction was performed using the Kapa HyperPlus Kits with 409 enzymatic fragmentation (Roche, Wilmington, MA). Fragmented gDNA were used to 410 perform end repair, A-tailing and adapter ligation following the manufacturer's 411 instruction. The indexed library construct was split into two fractions, one fraction was 412 used for WGS on NovaSeq6000 at PE2x150 cycles (Illumina, San Diego, CA) and 413 another fraction was normalized and pooled at 4 samples from tumor samples and 4 414 samples for germline samples. Pooled sample libraries were hybridized with SeqCap 415 EZ Human Exome v3.0 probes (Roche) for WES. The pooled, indexed and captured 416 final libraries were used to sequence on Illumina HiSeq4000 sequencer at 2x100 cycles 
417 pair-end reads. The raw sequencing reads in BCL format were processed through

418 bcl2fastq 2.19 (Illumina) for FASTQ conversion and demultiplexing for downstream data

419 analysis.

422 Processing of whole genome sequencing data

423 Overall, the median sequence coverage was 27.5X (range 15-55X; Supplemental

424 Data 1). Short insert paired-end reads/FASTQ files were aligned to the reference

425 human genome (GRCh37) using Burrows-Wheeler Aligner, BWA (v0.5.9). All samples

426 were uniformly analyzed by the whole-genome analysis bioinformatic pipeline

427 developed at the Memorial Sloan Kettering Cancer Center. ${ }^{24,26,45}$ Specifically: CaVEMan

428 was used for SNVs, indels were analyzed with Pindel, CNAs were explored by

429 Battenberg. To determine the tumor clonal architecture, and to model clusters of clonal

430 and subclonal point mutations, the Dirichlet process (DP) was applied. BRASS and

$431 \mathrm{JaBba}^{38}$ were used to detect SVs through discordantly mapping paired-end reads (large

432 inversions and deletions, translocations, and internal tandem duplication). Complex

433 events such as chromothripsis, chromoplexy, $d m$, bfb, templated insertions were

434 defined and validated after manual inspection as previously described. ${ }^{39,40,54,55}$ All SVs

435 not part of a complex event were define as single. Immunoglobulin VDJ, HCDR3, CRS,

436 and productivity were defined using lgcaller. ${ }^{50}$

438 Whole-Exome Sequencing 
Whole exome sequence data reads were aligned to the reference human

440 genome (GRCh37) using the Burrows-Wheeler Alignment tool (bwa mem v0.7.12).

441 PCR duplicate read removal, InDel realignment, fixing mates and base quality score

442 recalibration was applied to the aligned bams using PICARD tools or the Genome

443 Analysis Toolkit (GATK) according to GATK best practices. Samples in the selected

444 cohort had an average coverage of $76 X$ in tumors and $49 X$ in germline (range $15 X$ -

445 135X). The tumor purities were inferred using TITAN and ranged between 31-91\%.

446 WXS somatic calls were performed using CaVEMan for SNVs, Pindel for indels, and

447 Facets for CNA.

449 Mutational signatures

$450 \quad$ Analysis of SBS signatures was performed following our published workflow

451 based on three main steps: ${ }^{17}$ 1) de novo extraction; 2) assignment; and 3) fitting. ${ }^{17}$ For

452 the de novo extraction of mutational signatures we ran two independent algorithms;

453 SigProfiler and the hierarchical Dirichlet process (hdp). ${ }^{10,26}$ Next, each extracted

454 process was assigned to one or more mutational signatures included in the latest

455 COSMIC v3.2 catalog (https://cancer.sanger.ac.uk/signatures/sbs/). Lastly, mmsig, a

456 fitting algorithm designed for hematological cancers (DOI: 10.5281/zenodo.4541703),

457 was applied to accurately estimate the contribution of each mutational signature in each

458 sample. $^{29}$

$460 \quad$ Timing copy number and structural variant events.

461 The relative timing of each multi-chromosomal duplication event was estimated using 
462 the R package mol time (DOI: $10.5281 /$ zenodo.4542145). ${ }^{24,26}$ This approach allows the

463 estimation of the relative timing of acquisition of large $(>1 \mathrm{Mb})$ and clonal chromosomal

464 gains (3;1 or 4:1), trisomies (3:1), copy neutral LOH (2:0), and tetrasomies (4:1) using

465 the purity-corrected ratio between duplicated clonal clonal mutations (defined by DP).

466 SNVs were defined as duplicated or not according to the VAF corrected for the cancer

467 purity which was estimated combining purity estimates from both Battenberg and from

468 the SNV VAF density and distribution within clonal diploid regions. Overall, after

469 removing immunoglobulin loci and kataegis events, only CNA segments with more than

47050 clonal mutations were considered. ${ }^{24,26}$ Tetrasomies, with both alleles duplicated

471 (2:2), were removed given the impossibility of defining whether the two chromosomal

472 gains occurred in close temporal succession or not. ${ }^{24,26}$ Using this approach, we were

473 able to estimate the relative molecular time of each gained CNA segment allowing to

474 define if different chromosomal gains were acquired in the same or different time

475 windows. To define if different gains occurred in one single time window or in different

476 independent events we used multiple hierarchical clustering approach for each single

477 bootstrap solution (hclust $\mathrm{R}$ function; www.r-project.org) and we integrated the most

478 likely results with the Battenberg CNA changes over the time.

479 Next, we convert the relative molecular time estimate into an absolute estimate

480 (i.e., years of life) estimating the molecular time using only the SBS1 and SBS5

481 mutational burden within large chromosomal gains acquired in the same time window.

482 Applying this workflow we converted the SBS1 and SBS5-based molecular clock into an

483 absolute time for the acquisition of these events in each patient's life. ${ }^{23,25,26}$ Confidence

484 of intervals were generated by bootstrapping 1000 times the molecular time estimate. 
485 Similar to the relative molecular time approach, only multi-gain events with more than

48650 SBS1 and SBS5 clonal mutations were included.

487 To estimate the timing of loss-of-function events and the acquisition of distinct

488 SVs, we utilized two previously reported approaches: ${ }^{24}$ 1) we linked SV breakpoints to

489 the molecular time of chromosomal gains caused by the same SV; 2) we estimated the

490 relative time of SVs that occurred within large chromosomal gains based on the copy

491 number of the SV breakpoint. Approach (2) is based on the concept that any SV

492 involving a large gain can produce three different copy number scenarios: i) SV

493 associated loss occurred on the allele involved by the duplication before the duplication.

494 In this scenario, the deleted segment will not be duplicated creating a copy number

495 jump of at least 2 copies with the adjacent duplicated segments (e.g., 3:1 and 1:0).; ii)

496 the SV is responsible for a loss (or gain) on one of the two duplicated alleles (e.g., from

$4972: 1$ to $1: 1)$, suggesting that the SV event occurred after the chromosomal gain. iii) SV

498 causes the loss (or gain) of part of the minor allele creating a copy neutral LOH (e.g.,

499 from $2: 1$ to $2: 0 ;$. In this last scenario it is not possible to establish if the SV occurred

500 before or after the chromosomal duplication.

$501 \quad$ The recurrent mutations in driver genes chronological acquisition order was

502 estimated combining the Battenberg cancer cell fraction and pre/post gain data into a

503 Bradley Terry model as previously described, including just the earliest sample of each

504 patient. $^{24}$

505

506 Combined Immunophenotyping and FISH analysis. 
After immunophenotyping with mouse anti-CD30 antibody labelled with AMCA

508 (blue), the relevant tissue slides with coverslip are briefly reviewed and recorded under

509 fluorescence microscope for the intensity and quality of CD30 staining. After removing

510 the coverslip, the slides are washed in 2xSSC at room temperature for 5 minutes and

511 then fixed with $10 \%$ neutral buffered formalin for 20 minutes, followed by dehydrating

512 five minutes each in a series of $70 \%, 85 \%$ and $100 \%$ of alcohol. Slides then go through

513 the pretreatment for 10 minutes with $20 \mathrm{mM}$ Citrate Buffer/1\% NP-40 Mixture, $\mathrm{pH}$ 6.0-

514 6.5, followed by protease treatment at $40^{\circ} \mathrm{C}$ for 10-15 minutes (Abbott Molecular, Des

515 Plaines, IL), then by dehydrating 2 minutes each in a serios of $70 \%, 85 \%$ and $100 \%$ of

516 alcohol. Relevant FISH probes were selected based on WGS ploidy findings and

517 primarily were those used in clinical diagnosis tests, including CDK2 and CEP12, PDL1

518 and PDL2 and CEP9, and 19p and 19q, and EWSR1 (Abbott Molecular, Des Plaines, IL

519 and Genomic Empire, Buffalo, NY). These probes were labeled in spectrum orange,

520 green or aqua, respectively. After applying the FISH probes to the tissue areas, both

521 tissue and probes were co-denatured at $94^{\circ} \mathrm{C}$ for 7 minutes, and then incubated at $37^{\circ}$

522 C overnight, followed by post-hybridization washing in $2 \times S S C / 0.3 \% \mathrm{NP}-40$ at $77^{\circ} \mathrm{C}$ for

523 one minute. Tissue sections were counterstained with antifade medium without DAPI

524 (Vector Laboratories, Burlingame, CA).

525 The slides are evaluated under fluorescence microscope coupled with

526 appropriate filters for CD30 immunophenotype and for the relevant probes. Signal

527 analysis was performed in combination with tissue structure and cell morphology

528 correlation and was focused on the interested tissue areas with strong CD30 positive

529 cells only. The copy numbers of individual probes were counted in each case. 
531 Data analysis and statistics

$532 \quad$ Data analysis was carried out in $\mathrm{R}$ version 3.6.1. Standard statistical tests are

533 mentioned consecutively in the manuscript while more complex analyses are described

534 above. Wilcoxon rank-sum test between three groups was run using pairwise.wilcox.test

$535 \mathrm{R}$ function with all $\mathrm{p}$ value adjusted for FDR. All reported $\mathrm{p}$-values are two-sided, with a

536 significance threshold of $<0.05$.

538 Acknowledgements

539 This work was supported by the Children's Oncology Group, the Hartwell Foundation,

540 the Gant Family Foundation, the Sylvester Comprehensive Cancer Center NCI Core

541 Grant (P30 CA 240139), and the Memorial Sloan Kettering Cancer Center NCl Core

542 Grant (P30 CA 008748). F.M. is supported by the American Society of Hematology.

543 F.M. and O.L. are supported by the Riney Family Foundation. L.G.R is supported by the

$544 \mathrm{NIH}(\mathrm{K} 08 \mathrm{CA} 219473)$ and the Hartwell Foundation.

\section{Author contributions}

547 F.M., M.R., L.G.R. designed and supervised the study, collected and analyzed the data 548 and wrote the paper. M.I. J.Z.X, B.B, B.Z., F.A., K.H.M., K.W.E., M.U., F.H, W.Z., Q.G., 549 V.Y., O.L., C.M., E.C., T.H., O.E. collected and analyzed data. S.P., M.O., E.R., M.L., 550 G.W., C.F. and M.B. provided patient samples. All authors reviewed and edited the 551 manuscript. 


\section{Data availability}

555 The other already published data are deposited in the EGA and dbGap database

556 under the following accession numbers: EGAS00001001692: PCAWG cohort [

557 https://ega-archive.org/studies/EGAS00001001692 ]; EGAD00001003309, 67 WGS

558 raw data from 30 patients with $\mathrm{MM}$ and myeloma precursor conditions

559 [https://www.ebi.ac.uk/ega/datasets/EGAD00001003309]; phs000348.v2.p1, 22 WGS

560 raw data from patients with MM [https:/www.ncbi.nlm.nih.gov/projects/gap/cgi-

561 bin/study.cgi?study id=phs000348.v2.p1]. All these data are available under restricted

562 access, access can be obtained by contacting the public depository.

563 


\section{Figure Legends}

565 Figure 1. cHL mutational landscape. a) WGS mutational burden

566 comparison between $\mathrm{CHL}$ and other cancers included in the PCAWG $(n=2780)$ and in

567 MM ( $n=71)$ WGS studies. $\mathrm{cHL}$ is highlighted in red, other hematological cancers in blue.

568 The median of each tumor type is annotated with a red line. b) Whole genome

569 mutational burden comparison between Ped/AYA and older adults with cHL. p-value

570 was calculated using Wilcoxon rank-sum test. Boxplots show the median and

571 interquartile range. c) Heatmap summarizing the 25 mutated driver genes/hot spots

572 across $61 \mathrm{cHL}$ patient with available either WGS or WES data.

573

574 Figure 2. Copy number alterations (CNA) in cHL. a) Significant CNA GISTIC2.0

575 peaks and involved genes. b) Heatmap summarizing bi-allelic events and high level 576 gains ( $>6$ copies) involving driver genes extracted by GISTIC $(n=21)$ and by dndscv

577 ( $\mathrm{n}=27)$. c) Clonal/subclonal and duplicated/non-duplicated distribution of mutations in

578 driver genes. d) Chronological order of mutations in $25 \mathrm{cHL}$ driver genes. Two out of 25

579 driver mutations are not reported because they had no relative chronological 580 association with any other mutations in driver genes.

582 Figure 3. Mutational signatures in cHL. a) COSMICv.2 96-mutational profile of the 6 583 mutational signatures extracted and identified in $\mathrm{cHL}$. b) Contribution of mutational 584 signatures across $25 \mathrm{cHL}$ cases with available WGS data. c) Proportion of patients with 585 APOBEC activity across $\mathrm{CHL}$ and other cancers included in the PCAWG and in MM 586 WGS studies. cHL is highlighted in red, other hematological cancers in blue. d) 
APOBEC relative contribution among duplicated and not-duplicated mutations within

588 large chromosomal gains.

590 Figure 4. Molecular time and clock in cHL. a-b) Two examples showing molecular

591 time estimates for two cases with multiple gains. Left, the molecular time (blue dots)

592 estimated for each clonal gain and copy-neutral loss of heterozygosity with more than

59350 clonal SNVs. Red dots represent the molecular time of a second gain occurring on a

594 previous one. The dashed green line divides the two independent time windows in

595 which different chromosomal gains were acquired ( $1^{\text {st }} \mathrm{t}$ : first time window; 2 st $t$ : second

596 time window. Right, standard copy number profile of $2 \mathrm{cHL}$ cases. Horizontal purple

597 and blue lines represent the total copy number and minor allele, respectively. c) Median

598 molecular time estimates for chromosomal gains across each chromosome in cHL.

599 Each chromosome was divided into $10 \mathrm{mb}$ bins. Each green dot reflects the median

600 molecular time across different patients with the gained bin. $\mathrm{Cl}$ were generated using

601 the median of $\mathrm{Cl}$ molecular time estimate for each bin. Horizontal red line reflects

602 GISTIC large amplifications. The blue/yellow plot represents the distribution of

603 chromosomal gains acquired within either the first- or second-time window for each loci.

604 d) Linear regression showing the association between age and SBS1/SBS5 mutational

605 burden in $\mathrm{cHL}-\mathrm{e}$ ) Estimated patient age at the first (blue) and second (green) multi-gain

606 event with $95 \%$ Cls. Red dots represent age at sample collection. Case numbers written

607 in red represent cases collected at relapse.

608 
609 Figure 5. SV and complex event in cHL. a) Heatmap showing distribution of complex

610 events across the $25 \mathrm{cHL}$ cases evaluated by WGS. dm: double minutes, bfb:

611 breakage-fusion-bridge, tic: templated insertion. b-c) Example of complex events

612 involving key $\mathrm{cHL}$ drivers. d-e) Example of chromothripsis event in which it was possible

613 to estimate the molecular time. Case IID_H198448 had an intrachromosomal

614 chromothripsis event responsible for multiple large/intermediate chromosomal gain on

615 chromosome $17(\mathbf{5 d})$. The molecular time of this event was similar to other gains

616 acquired in the first/earliest-time window (5e). Based on this link we could estimate that

617 that the chromothripsis event was acquired together with the other chromosomal gains

618 (WGD) and approximately 10 years before the diagnosis (95\% Cl 8-13). f-g) Examples

619 of two chromothripsis events that occurred before WGD. The two chromothripsis

620 events were each responsible for a multiple copy number jump from 2:2 to 2:0. In d), f)

621 and $\mathbf{g}$ ), the black and dashed yellow horizontal line represent the total number copy

622 number and the minor allele, respectively. The blue, red, green, and black vertical lines

623 represent inversion, deletion, tandem duplication, and translocation respectively. The

624 partner of each translocation is reported on the top of the vertical black line.

626 Figure 6. AID and SHM in cHL. a) Mutation density across the immunoglobulin loci. b)

627 96-mutational profile of all mutations occurred within the Ig loci. c) 96-mutational profile

628 of all mutations occurred within known AID off-targets. The profile in b) and c) is

629 identical to SBS84 (see Figure 3a). d) SBS9 and SBS84 distribution and activity across

630 different lymphoproliferative disorders according to Maura et al. $^{17}$ and Alexandrov et

631 al. $^{10}$ ). ALL: acute lymphoblastic leukemia; BL: Burkitt lymphoma; DLBCL: diffuse large 
632 B-cell lymphoma; FL: follicular lymphoma; MM: multiple myeloma; M-CLL: mutated

633 IGHV chronic lymphocytic leukemia; U-CLL: unmutated IGHV chronic lymphocytic

634 leukemia; HL: Hodgkin lymphoma. Each mutational signature activity is highlighted in

635 red. e) Heatmap summarizing BCR productivity in our cohort of 25 cases with WGS. f)

636 Proportion of duplicated and non-duplicated mutations within immunoglobulin loci and

637 AID-off target involved by chromosomal duplications occurring within the earliest

638 molecular time window. The last two bars on the right represent the expected proportion

639 in the situation where the mutation was acquired before or after the chromosomal gain.

640

641 Figure 7. Summary of the proposed $\mathrm{cHL}$ germinal center-based pathogenetic 642 model. 


\section{References}

6471 Mathas, S., Hartmann, S. \& Kuppers, R. Hodgkin lymphoma: Pathology and

$648 \quad$ biology. Semin Hematol 53, 139-147, doi:10.1053/j.seminhematol.2016.05.007

6502 Reichel, J. et al. Flow sorting and exome sequencing reveal the oncogenome of

3 Spina, V. et al. Circulating tumor DNA reveals genetics, clonal evolution, and residual disease in classical Hodgkin lymphoma. Blood 131, 2413-2425, doi:10.1182/blood-2017-11-812073 (2018).

4 Tiacci, E. et al. Pervasive mutations of JAK-STAT pathway genes in classical Hodgkin lymphoma. Blood 131, 2454-2465, doi:10.1182/blood-2017-11-814913 (2018).

5 Wienand, K. et al. Genomic analyses of flow-sorted Hodgkin Reed-Sternberg cells reveal complementary mechanisms of immune evasion. Blood Adv 3, 40654080, doi:10.1182/bloodadvances.2019001012 (2019).

6 Consortium, I. T. P.-C. A. O. W. G. Pan-cancer analysis of whole genomes. Nature 578, 82-93, doi:10.1038/s41586-020-1969-6 (2020).

7 Reichel, J. B. et al. Flow-sorting and Exome Sequencing of the Reed-Sternberg Cells of Classical Hodgkin Lymphoma. J Vis Exp, doi:10.3791/54399 (2017).

8 Tanaka, N. et al. Sequencing artifacts derived from a library preparation method using enzymatic fragmentation. PLoS One 15, e0227427, doi:10.1371/journal.pone.0227427 (2020).

9 Lee-Six, H. et al. The landscape of somatic mutation in normal 1 colorectal epithelial cells. bioRxiv (2018).

10 Alexandrov, L. B. et al. The repertoire of mutational signatures in human cancer. Nature 578, 94-101, doi:10.1038/s41586-020-1943-3 (2020).

11 Martincorena, I. et al. Universal Patterns of Selection in Cancer and Somatic Tissues. Cell 173, 1823, doi:10.1016/j.cell.2018.06.001 (2018).

12 Mottok, A. et al. Inactivating SOCS1 mutations are caused by aberrant somatic hypermutation and restricted to a subset of B-cell lymphoma entities. Blood 114, 4503-4506, doi:10.1182/blood-2009-06-225839 (2009).

13 Balinas-Gavira, C. et al. Frequent mutations in the amino-terminal domain of BCL7A impair its tumor suppressor role in DLBCL. Leukemia 34, 2722-2735, doi:10.1038/s41375-020-0919-5 (2020).

14 Panea, R. I. et al. The whole-genome landscape of Burkitt lymphoma subtypes. Blood 134, 1598-1607, doi:10.1182/blood.2019001880 (2019).

15 Trengove, M. C. \& Ward, A. C. SOCS proteins in development and disease. Am $J$ Clin Exp Immunol 2, 1-29 (2013).

16 Kasar, S. et al. Whole-genome sequencing reveals activation-induced cytidine deaminase signatures during indolent chronic lymphocytic leukaemia evolution. Nat Commun 6, 8866, doi:10.1038/ncomms9866 (2015).

17 Maura, F. et al. A practical guide for mutational signature analysis in 11037-8 (2019). 
69118 Maura, F. et al. Role of AID in the temporal pattern of acquisition of driver

692

693

694

695

696

697

698

699

700

701

702

703

704

705

706

707

708

709

710

711

712

713

714

715

716

717

718

719

720

721

722

723

724

725

726

727

728

729

730

731

732

733

734

735 mutations in multiple myeloma. Leukemia 34, 1476-1480, doi:10.1038/s41375019-0689-0 (2020).

19 Martin-Subero, J. I. et al. Recurrent involvement of the REL and BCL11A loci in classical Hodgkin lymphoma. Blood 99, 1474-1477, doi:10.1182/blood.v99.4.1474 (2002).

20 Martin-Subero, J. I. et al. Chromosomal breakpoints affecting immunoglobulin loci are recurrent in Hodgkin and Reed-Sternberg cells of classical Hodgkin lymphoma. Cancer Res 66, 10332-10338, doi:10.1158/0008-5472.CAN-06-1992 (2006).

21 Roemer, M. G. et al. PD-L1 and PD-L2 Genetic Alterations Define Classical Hodgkin Lymphoma and Predict Outcome. J Clin Oncol 34, 2690-2697, doi:10.1200/JCO.2016.66.4482 (2016).

22 Mermel, C. H. et al. GISTIC2.0 facilitates sensitive and confident localization of the targets of focal somatic copy-number alteration in human cancers. Genome Biol 12, R41, doi:10.1186/gb-2011-12-4-r41 (2011).

23 Gerstung, M. et al. The evolutionary history of 2,658 cancers. Nature 578, $122-$ 128, doi:10.1038/s41586-019-1907-7 (2020).

24 Maura, F. et al. Genomic landscape and chronological reconstruction of driver events in multiple myeloma. Nat Commun 10, 3835, doi:10.1038/s41467-01911680-1 (2019).

25 Mitchell, T. J. et al. Timing the Landmark Events in the Evolution of Clear Cell Renal Cell Cancer: TRACERx Renal. Cell 173, 611-623 e617, doi:10.1016/j.cell.2018.02.020 (2018).

26 Rustad, E. H. et al. Timing the initiation of multiple myeloma. Nat Commun 11, 1917, doi:10.1038/s41467-020-15740-9 (2020).

27 Degasperi, A. et al. A practical framework and online tool for mutational signature analyses show inter-tissue variation and driver dependencies. Nat Cancer $\mathbf{1}$, 249-263, doi:10.1038/s43018-020-0027-5 (2020).

28 Koh, G., Degasperi, A., Zou, X., Momen, S. \& Nik-Zainal, S. Mutational signatures: emerging concepts, caveats and clinical applications. Nat Rev Cancer, doi:10.1038/s41568-021-00377-7 (2021).

29 Rustad, E. H. et al. mmsig: a fitting approach to accurately identify somatic mutational signatures in hematological malignancies. Commun Biol 4, 424, doi:10.1038/s42003-021-01938-0 (2021).

30 Alexandrov, L. B. et al. Clock-like mutational processes in human somatic cells. Nat Genet 47, 1402-1407, doi:10.1038/ng.3441 (2015).

31 Chan, K. et al. An APOBEC3A hypermutation signature is distinguishable from the signature of background mutagenesis by APOBEC3B in human cancers. Nat Genet 47, 1067-1072, doi:10.1038/ng.3378 (2015).

32 Petljak, M. \& Maciejowski, J. Molecular origins of APOBEC-associated mutations in cancer. DNA Repair (Amst) 94, 102905, doi:10.1016/j.dnarep.2020.102905 (2020).

33 Lee-Six, H. et al. The landscape of somatic mutation in normal colorectal epithelial cells. Nature 574, 532-537, doi:10.1038/s41586-019-1672-7 (2019). 
73634 Maura, F. et al. The mutagenic impact of melphalan in multiple myeloma. Leukemia 35, 2145-2150, doi:10.1038/s41375-021-01293-3 (2021).

35 Pich, O. et al. The evolution of hematopoietic cells under cancer therapy. Nat Commun 12, 4803, doi:10.1038/s41467-021-24858-3 (2021). 1740, doi:10.1038/s41588-019-0525-5 (2019).

37 Landau, H. J. et al. Accelerated single cell seeding in relapsed multiple myeloma. Nat Commun 11, 3617, doi:10.1038/s41467-020-17459-z (2020).

38 Hadi, K. et al. Distinct Classes of Complex Structural Variation Uncovered across Thousands of Cancer Genome Graphs. Cell 183, 197-210 e132, doi:10.1016/j.cell.2020.08.006 (2020).

$39 \mathrm{Li}, \mathrm{Y}$. et al. Patterns of somatic structural variation in human cancer genomes. Nature 578, 112-121, doi:10.1038/s41586-019-1913-9 (2020).

40 Rustad, E. H. et al. Revealing the impact of structural variants in multiple myeloma. Blood Cancer Discov 1, 258-273, doi:10.1158/2643-3230.bcd-20-0132 (2020).

41 Kanzler, H., Kuppers, R., Hansmann, M. L. \& Rajewsky, K. Hodgkin and ReedSternberg cells in Hodgkin's disease represent the outgrowth of a dominant tumor clone derived from (crippled) germinal center B cells. J Exp Med 184, 1495-1505, doi:10.1084/jem.184.4.1495 (1996).

42 Kuppers, R. et al. Hodgkin disease: Hodgkin and Reed-Sternberg cells picked from histological sections show clonal immunoglobulin gene rearrangements and appear to be derived from B cells at various stages of development. Proc Natl Acad Sci U S A 91, 10962-10966, doi:10.1073/pnas.91.23.10962 (1994).

43 Kuppers, R. et al. Hodgkin's disease: clonal lg gene rearrangements in Hodgkin and Reed-Sternberg cells picked from histological sections. Ann N Y Acad Sci 764, 523-524, doi:10.1111/j.1749-6632.1995.tb55877.x (1995).

44 Kuppers, R., Zhao, M., Hansmann, M. L. \& Rajewsky, K. Tracing B cell development in human germinal centres by molecular analysis of single cells picked from histological sections. EMBO J 12, 4955-4967 (1993).

45 Bolli , F. et al. Genomic patterns of progression in smoldering multiple myeloma. Nat Commun 9(1), doi:10.1038/s41467-018-05058-y (2018).

46 Hubschmann, D. et al. Mutational mechanisms shaping the coding and noncoding genome of germinal center derived B-cell lymphomas. Leukemia 35 , 2002-2016, doi:10.1038/s41375-021-01251-z (2021).

47 Puente, X. S. et al. Non-coding recurrent mutations in chronic lymphocytic leukaemia. Nature 526, 519-524, doi:10.1038/nature14666 (2015).

48 Oben, B. et al. Whole-genome sequencing reveals progressive versus stable myeloma precursor conditions as two distinct entities. Nat Commun 12, 1861, doi:10.1038/s41467-021-22140-0 (2021).

49 Waanders, E. et al. Mutational landscape and patterns of clonal evolution in relapsed pediatric acute lymphoblastic leukemia. Blood Cancer Discov 1, 96-111, doi:10.1158/0008-5472.BCD-19-0041 (2020).

50 Nadeu, F. et al. IgCaller for reconstructing immunoglobulin gene rearrangements and oncogenic translocations from whole-genome sequencing in lymphoid neoplasms. Nat Commun 11, 3390, doi:10.1038/s41467-020-17095-7 (2020). 
78251 Paulsson, K. et al. The genomic landscape of high hyperdiploid childhood acute 783 lymphoblastic leukemia. Nat Genet 47, 672-676, doi:10.1038/ng.3301 (2015).

78452 Machado, E. et al. Genome-wide mutational signatures of immunological

785 diversification in normal lymphocytes. bioRxiv,

786 doi:https://doi.org/10.1101/2021.04.29.441939 (2021).

78753 Zhang, L. et al. Single-cell whole-genome sequencing reveals the functional landscape of somatic mutations in B lymphocytes across the human lifespan. Proc Natl Acad Sci U S A 116, 9014-9019, doi:10.1073/pnas.1902510116 (2019).

54 Korbel, J. O. \& Campbell, P. J. Criteria for inference of chromothripsis in cancer

796

55 Maciejowski, J. et al. APOBEC3-dependent kataegis and TREX1-driven chromothripsis during telomere crisis. Nat Genet 52, 884-890, doi:10.1038/s41588-020-0667-5 (2020). 

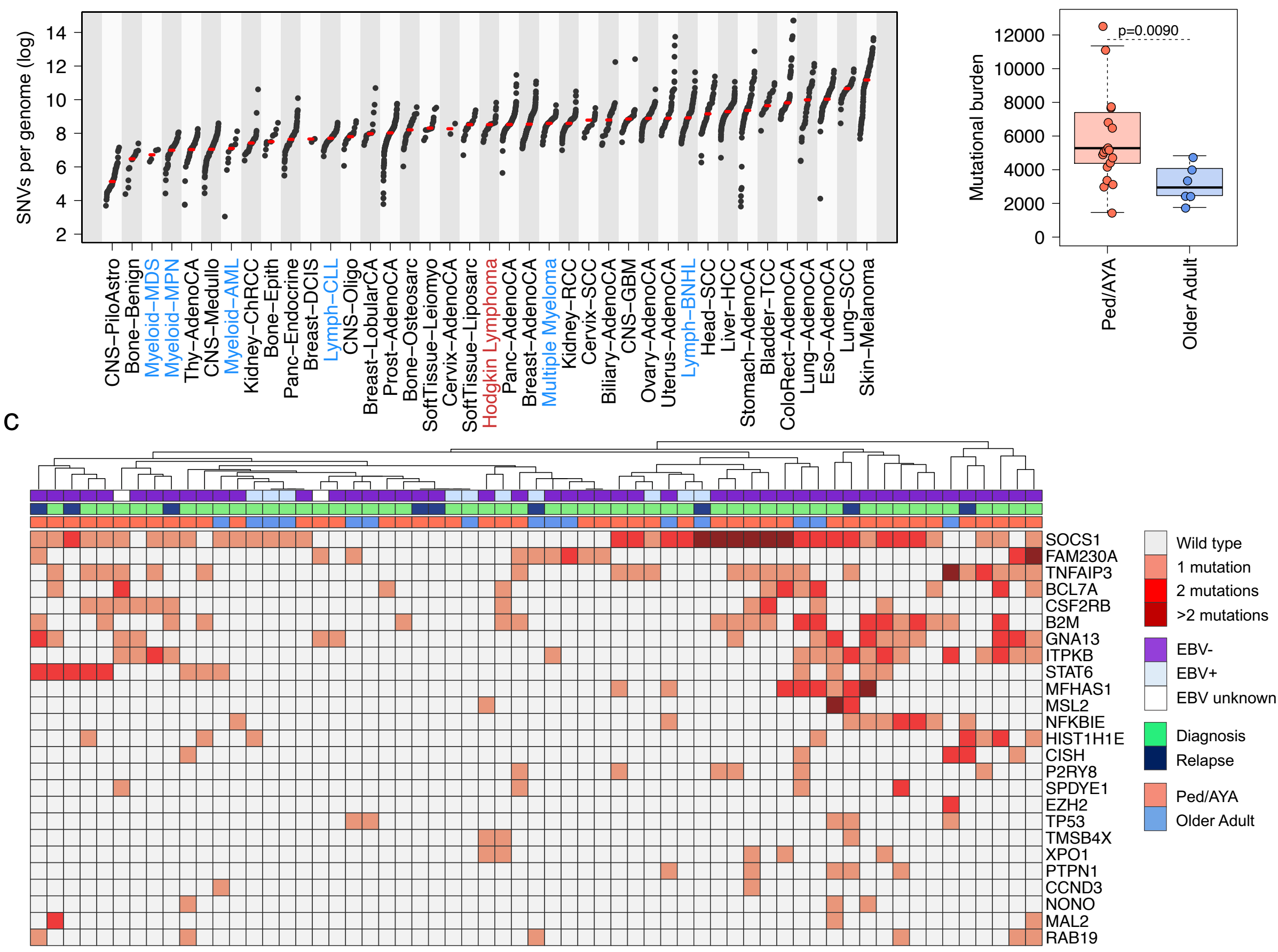

Figure 1 
a

\section{GISTIC amplification}

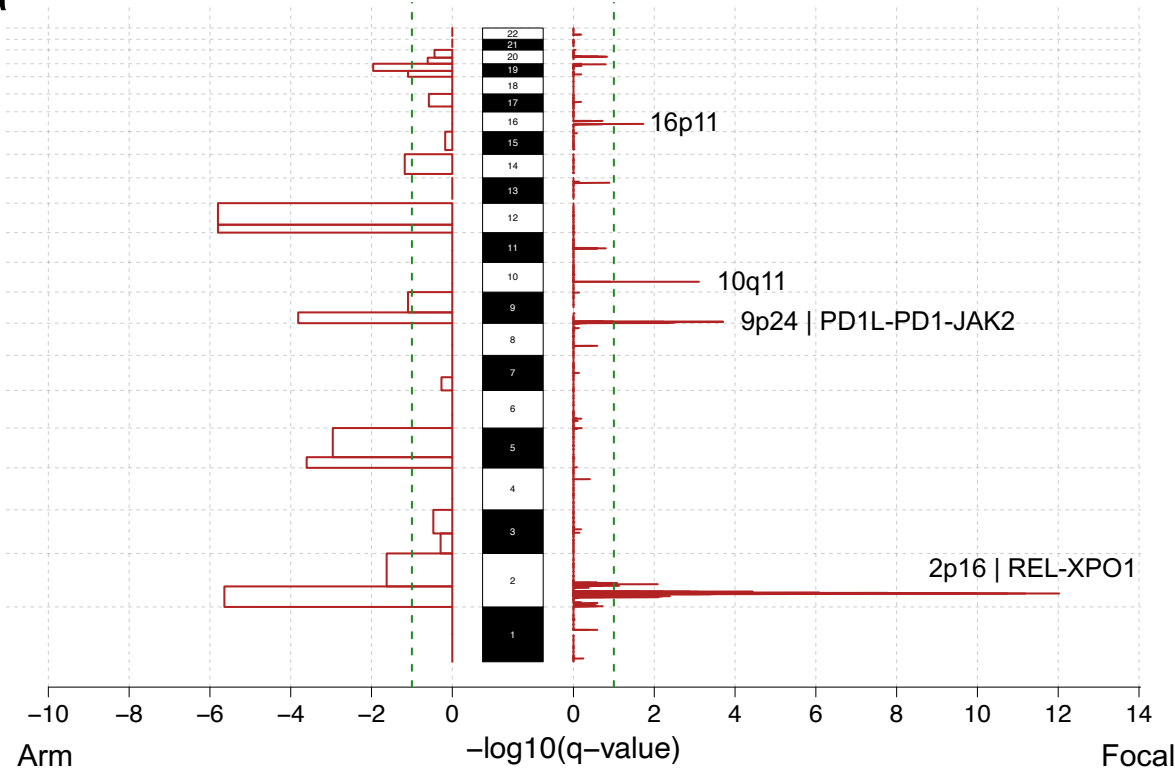

GISTIC deletion

22q11 | IGL

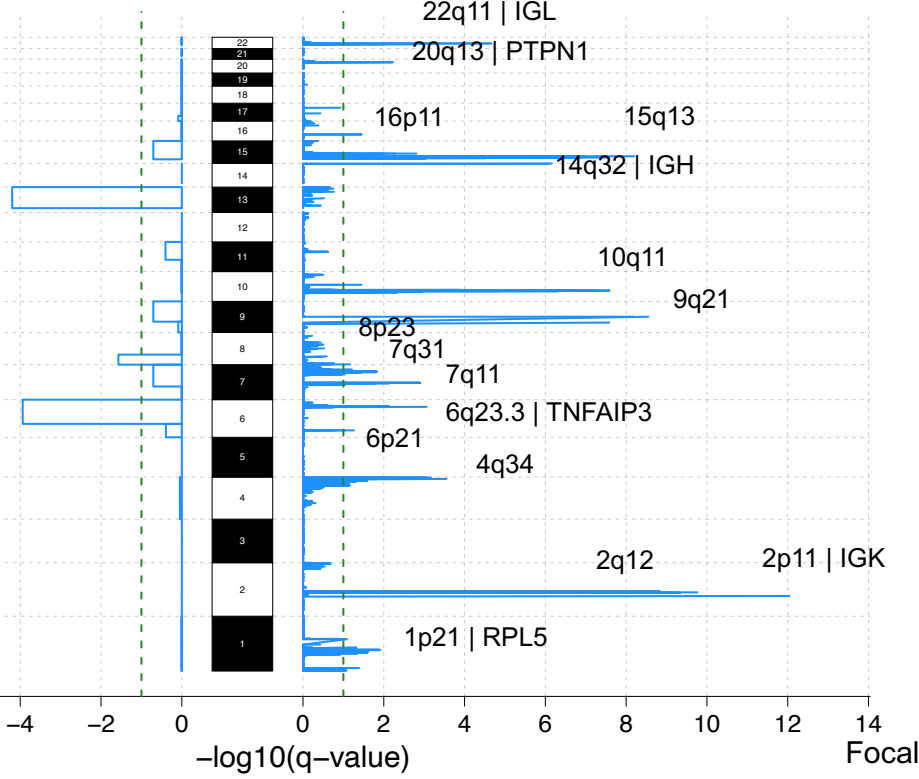

b

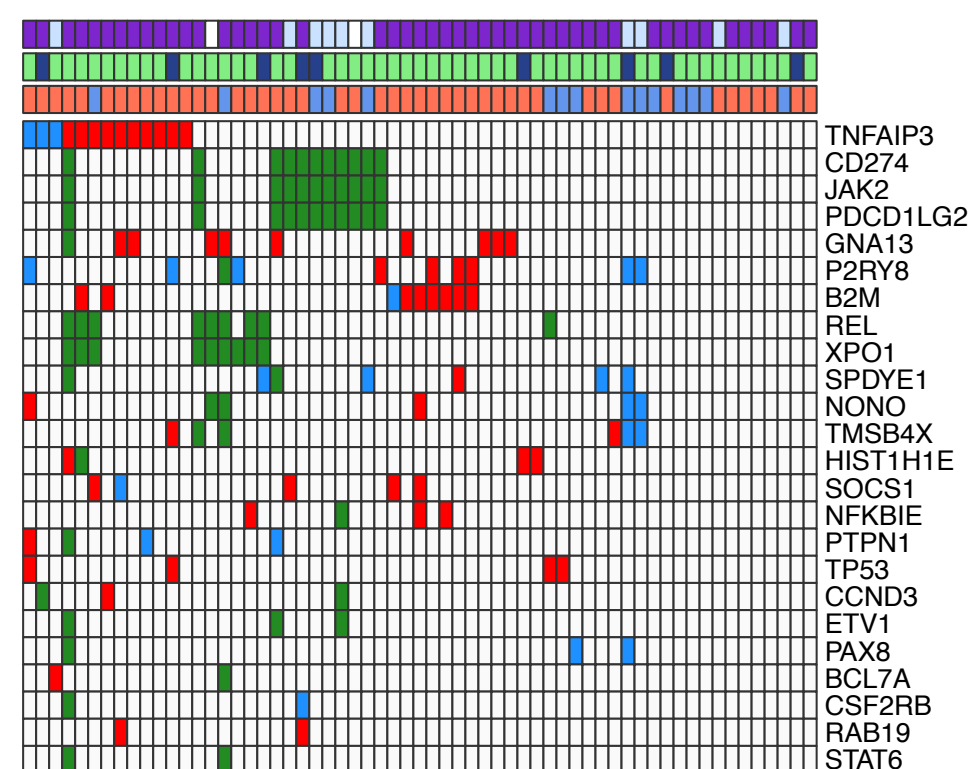

C

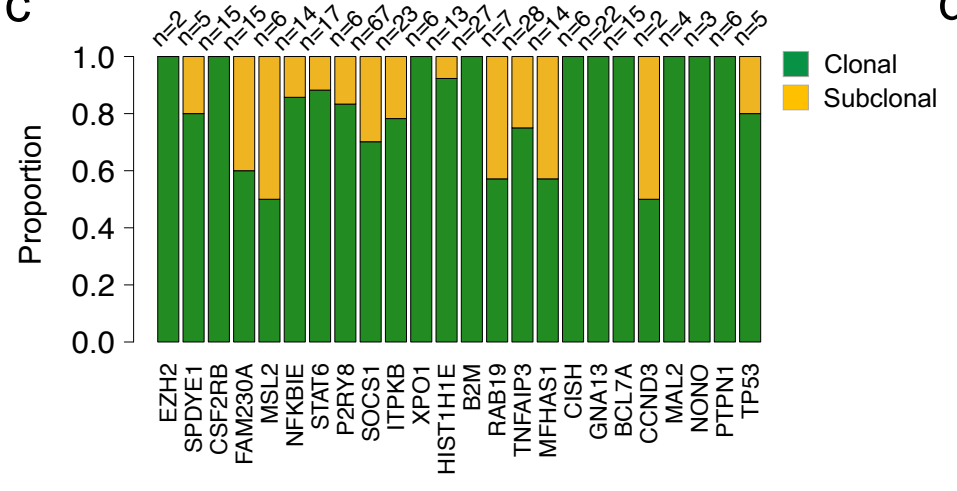

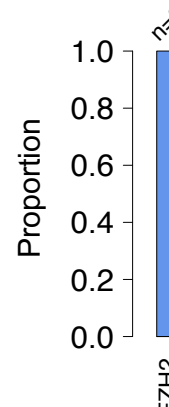

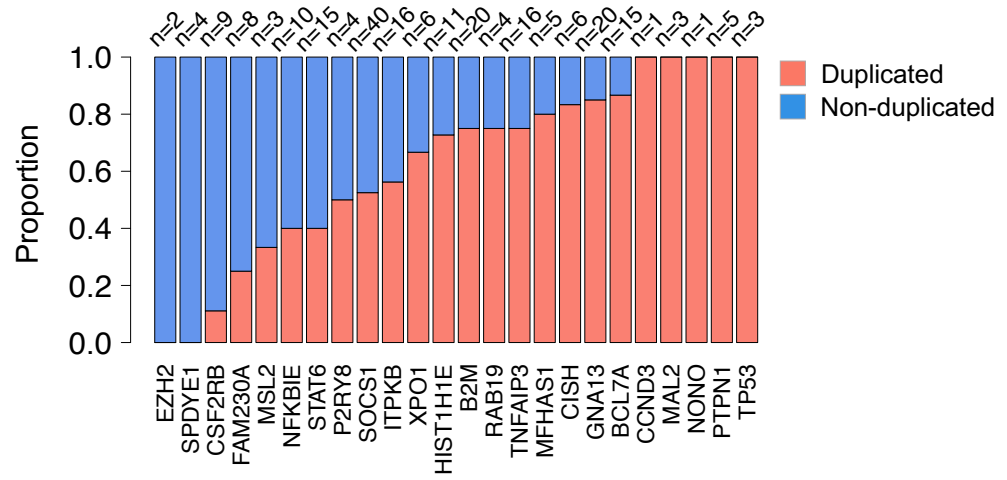

d

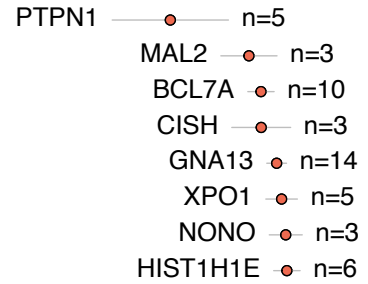

HIST1H1E ค $\mathrm{n}=6$

$B 2 M \circ n=19$

STAT6 ○ $\mathrm{n}=11$

TP53 $-\mathrm{n}=4$

RAB19 $\circ \mathrm{n}=6$

ITPKB ○ $\mathrm{n}=16$

CSF2RB ○ $n=11$

TNFAIP3 $\circ n=21$

SOCS1 1 $n=32$

NFKBIE ค $\mathrm{n}=7$

MFHAS1 $\circ \mathrm{n}=8$

P2RY8 $\circ \mathrm{n}=6$

MSL2 ○ $n=3$

FAM230A - $\mathrm{n}=7$

TMSB4X $-\mathrm{n}=3$

SPDYE1 - $\mathrm{n}=4$

$\begin{array}{llllll}-2 & 0 & 2 & 4 & 6 & 8\end{array}$

Figure 2 
a

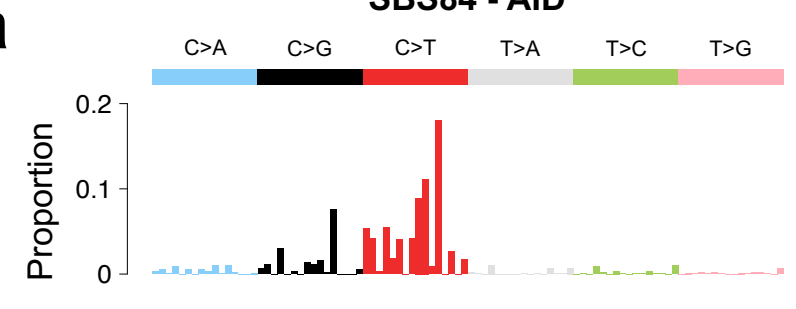

SBS2 - APOBEC

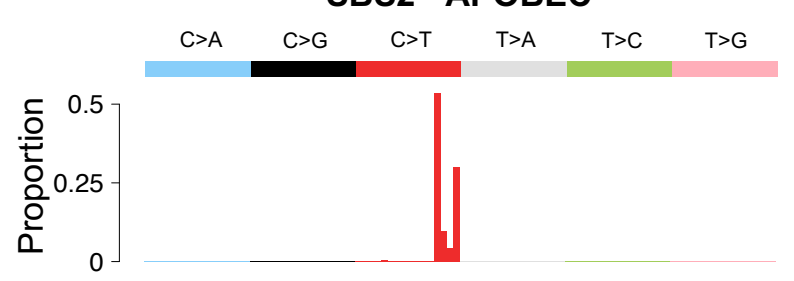

b

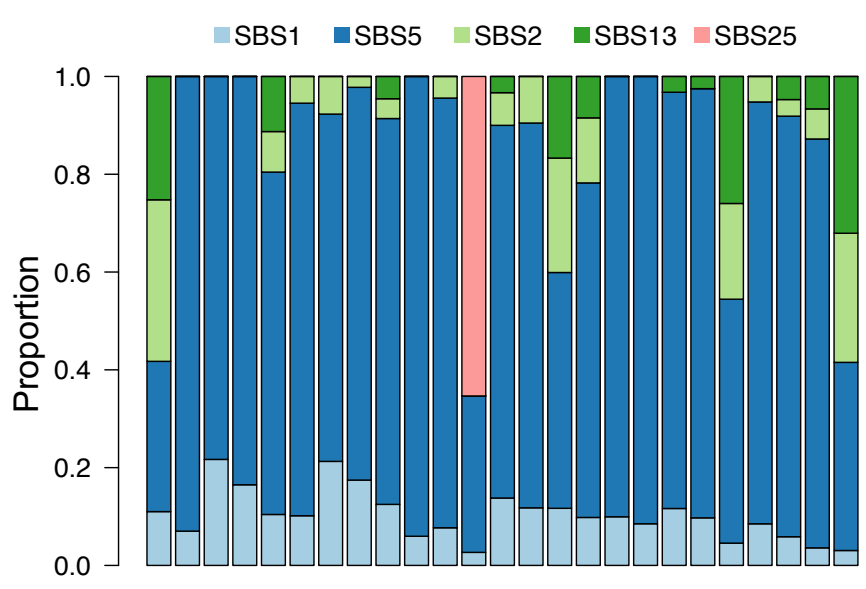

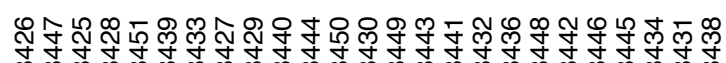

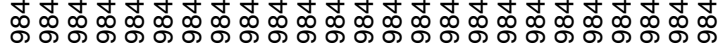

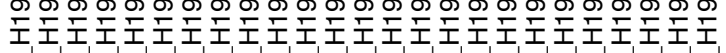

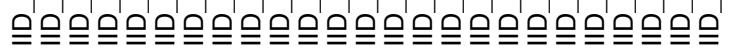

SBS1 - Aging
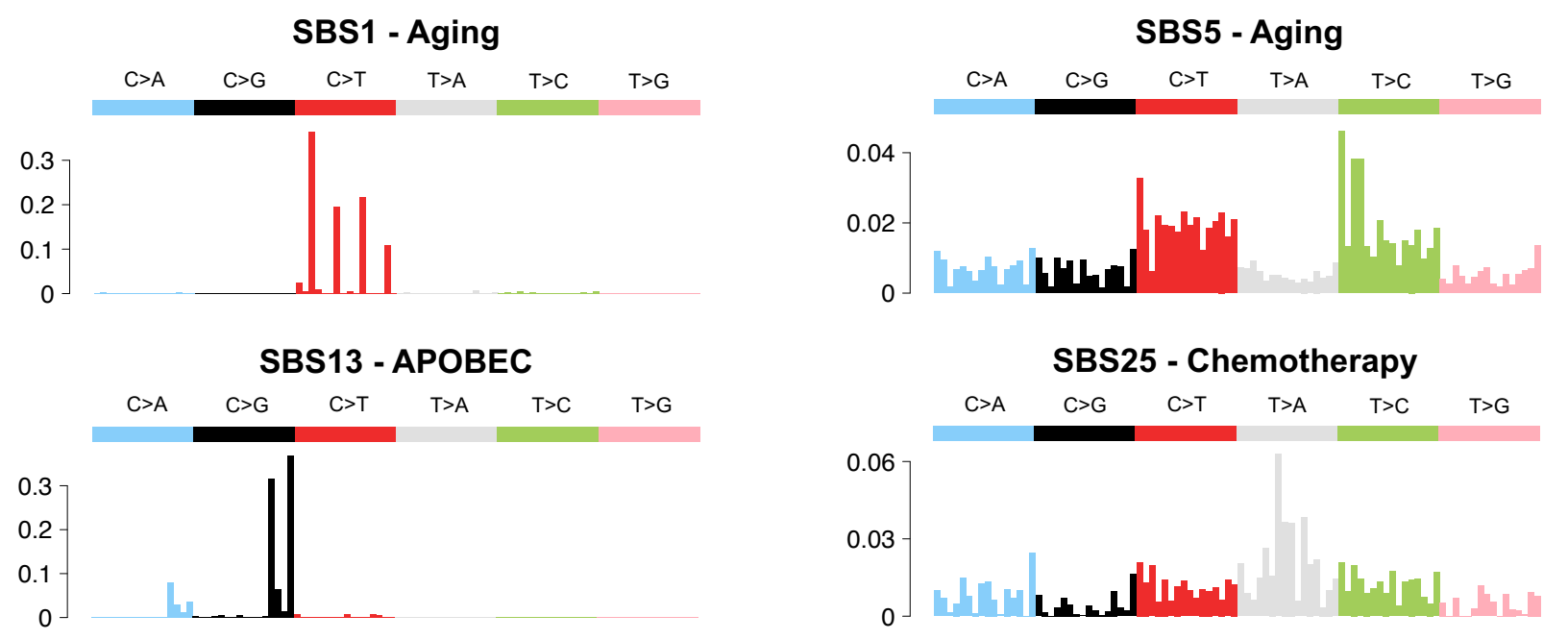

C
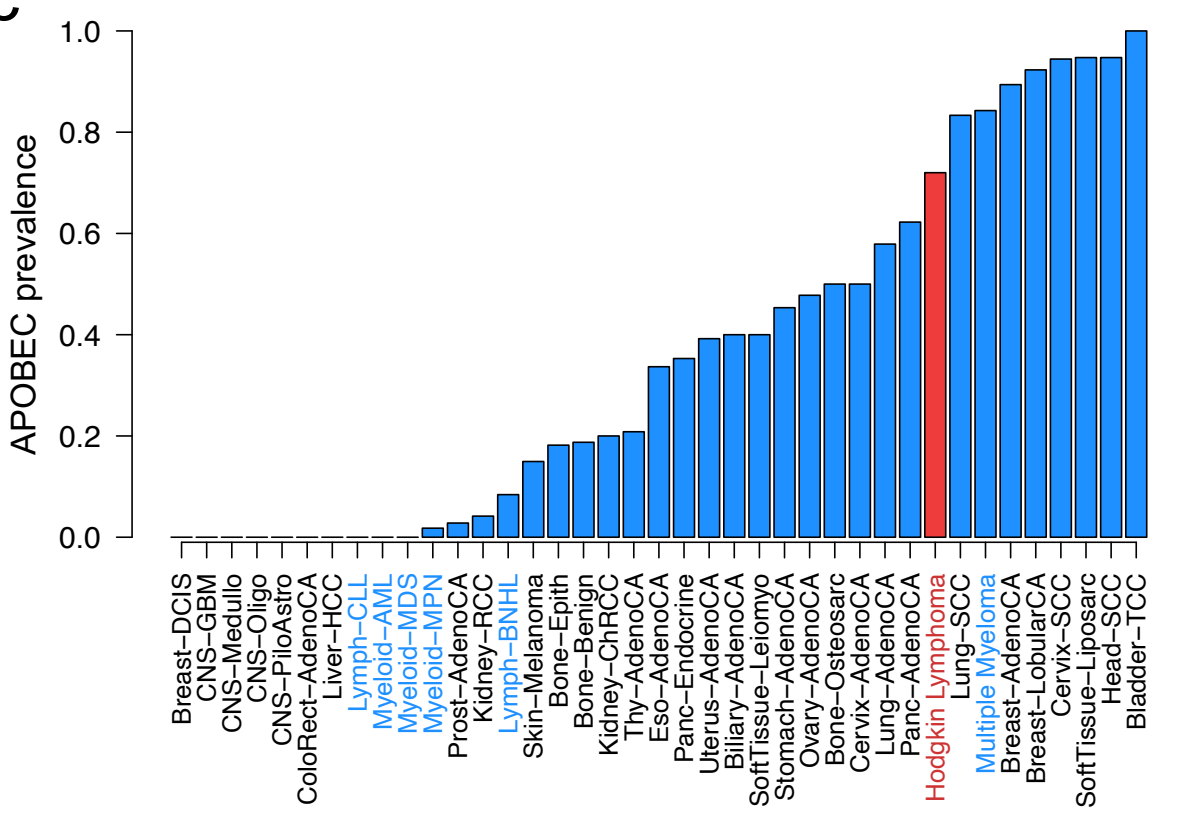

d

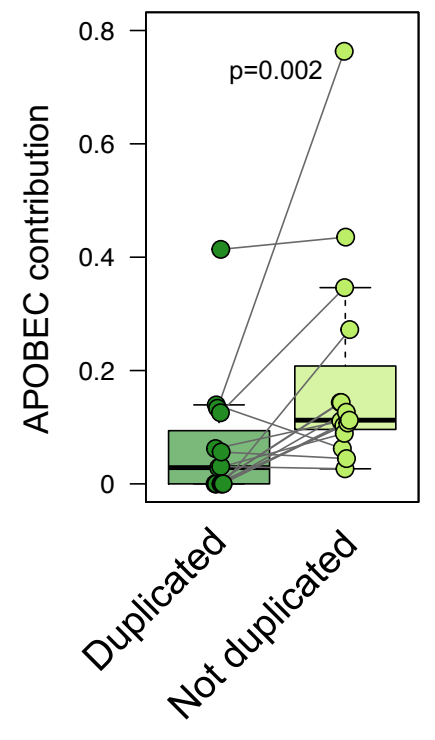

Figure 3 


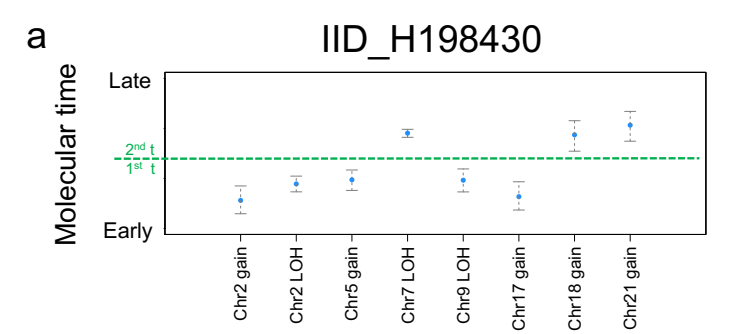

b

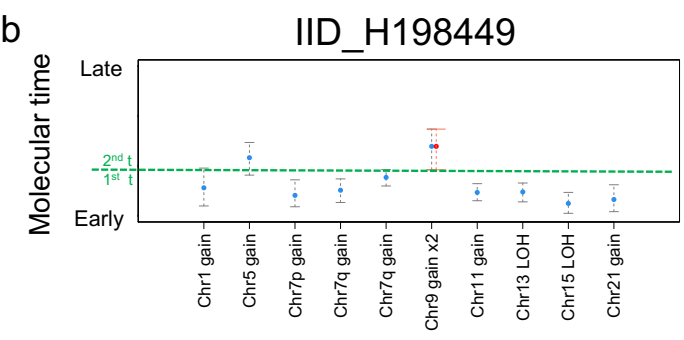

C

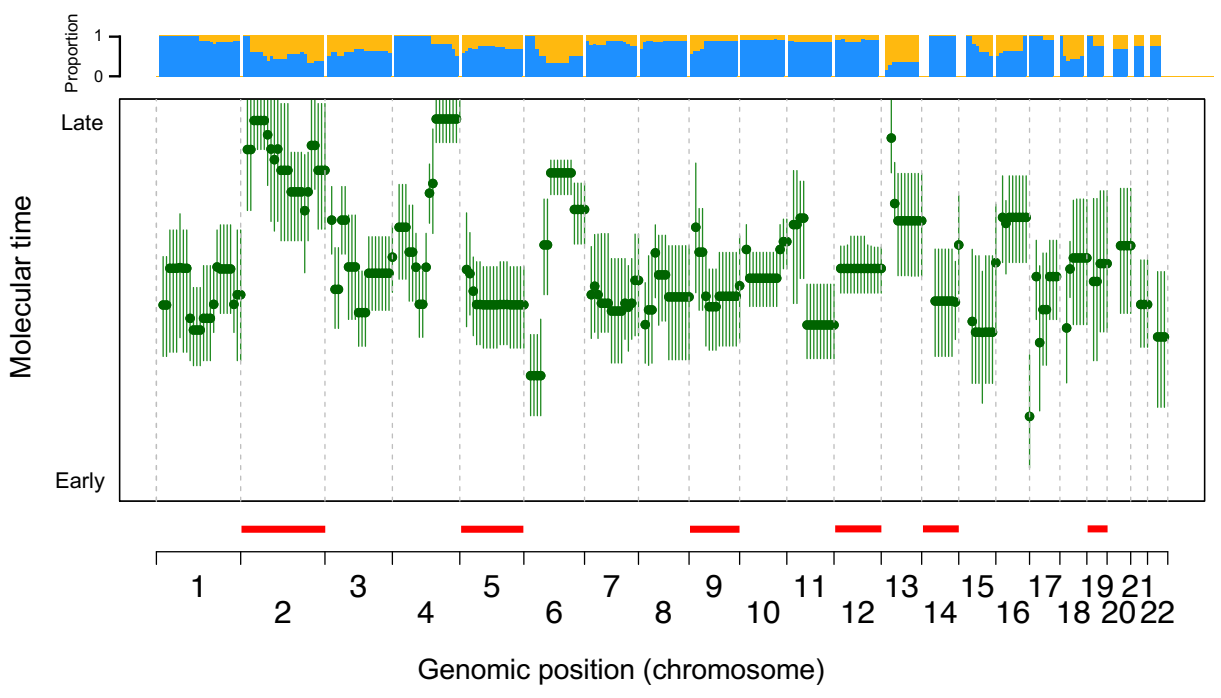

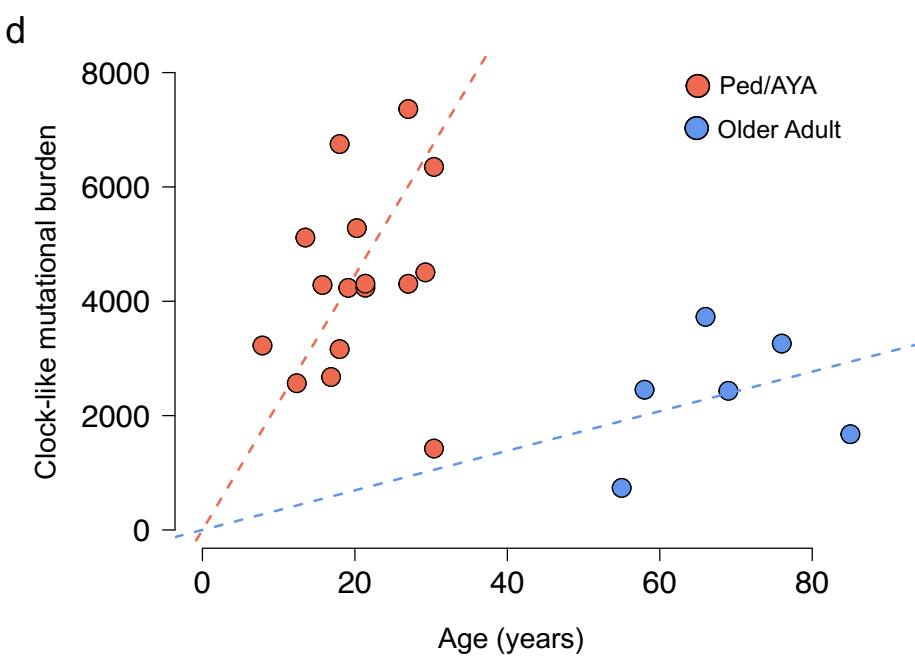

e

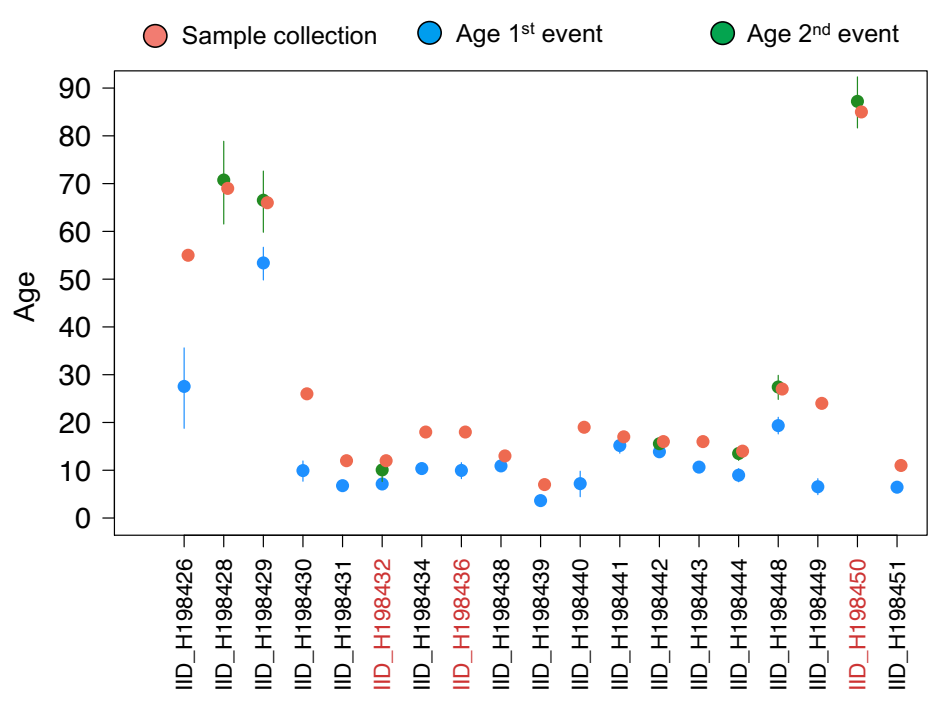

\section{Figure 4}




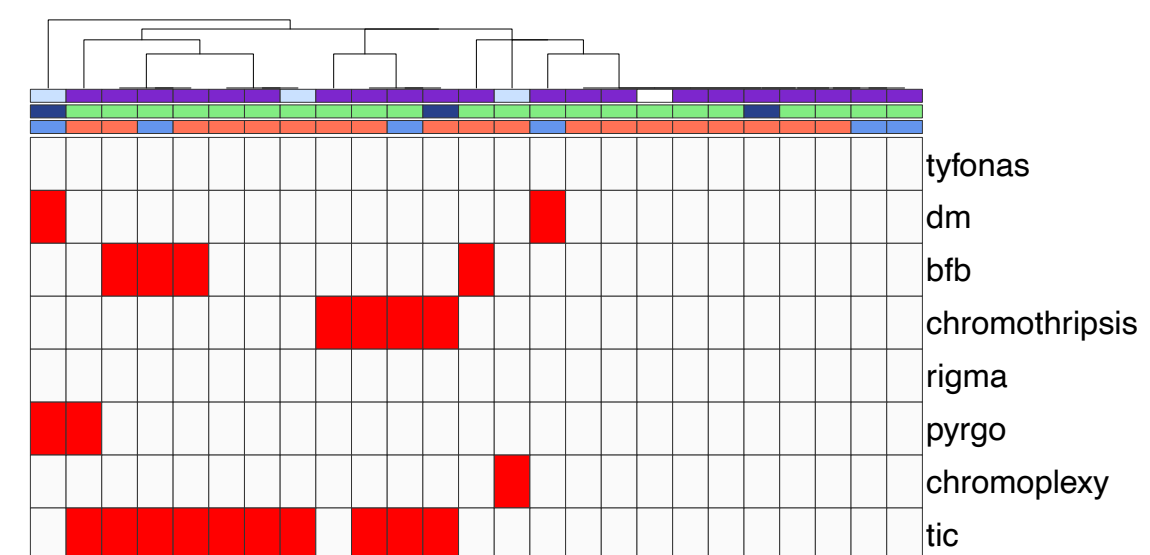

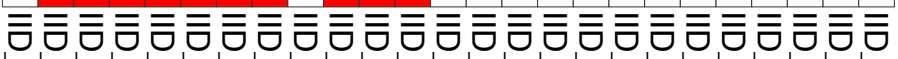

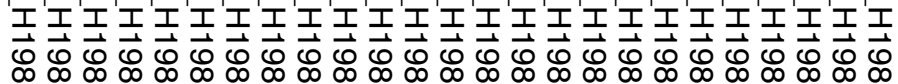

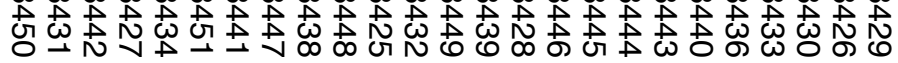

\begin{tabular}{|l|l|l|l|l}
\hline $\begin{array}{l}\text { Ped/AYA } \\
\text { Older Adult }\end{array}$ & $\begin{array}{l}\text { Diagnosis } \\
\text { Relapse }\end{array}$ & $\begin{array}{l}\text { EBV- } \\
\text { EBV+ } \\
\text { EBV unknown }\end{array}$ & Present \\
& &
\end{tabular}

d

IID_H198448 - Chromosome 17

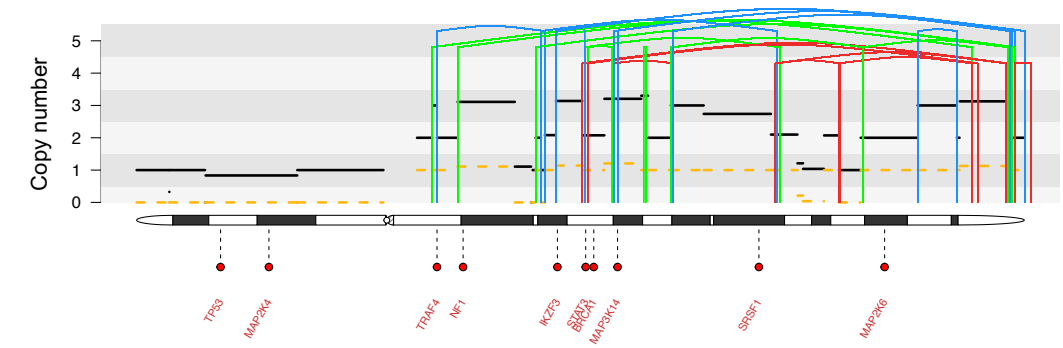

f

IID_H198438 - Chromosome 20

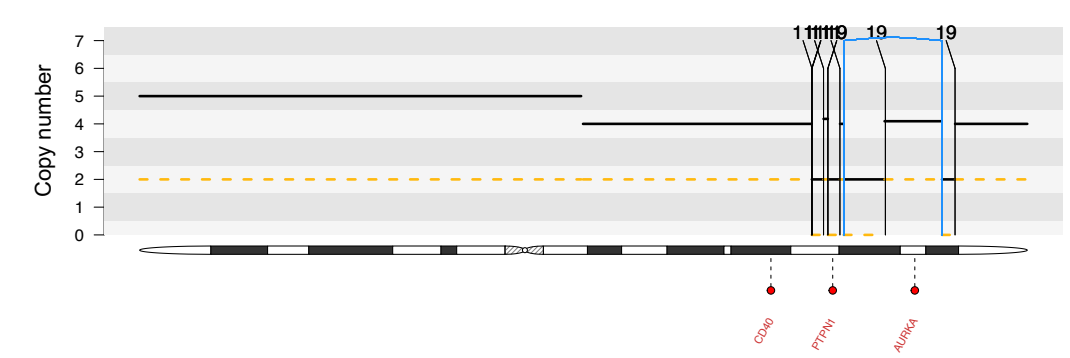

b

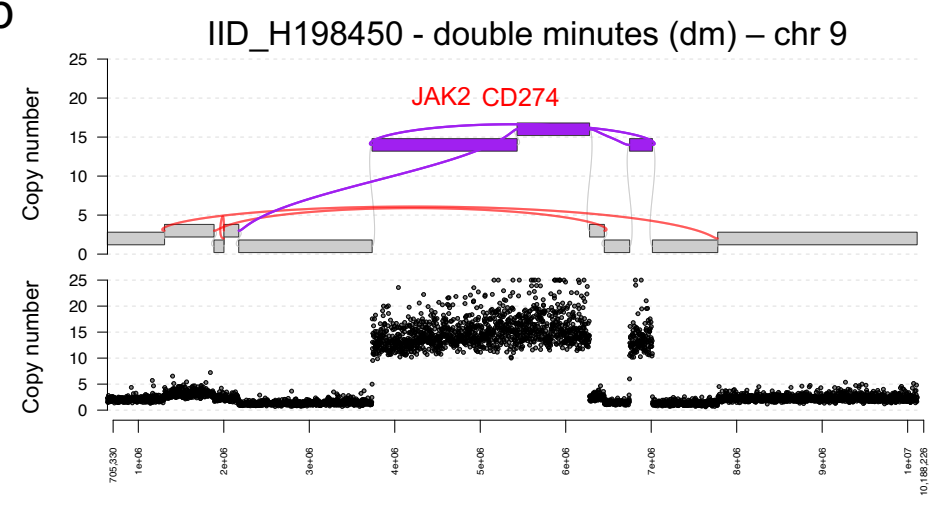

C

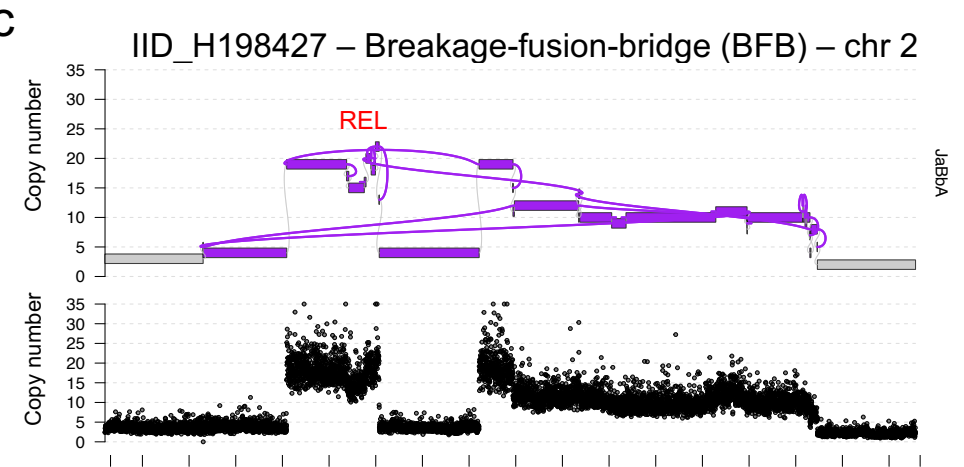

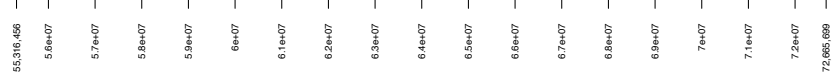

e

IID_H198448

9

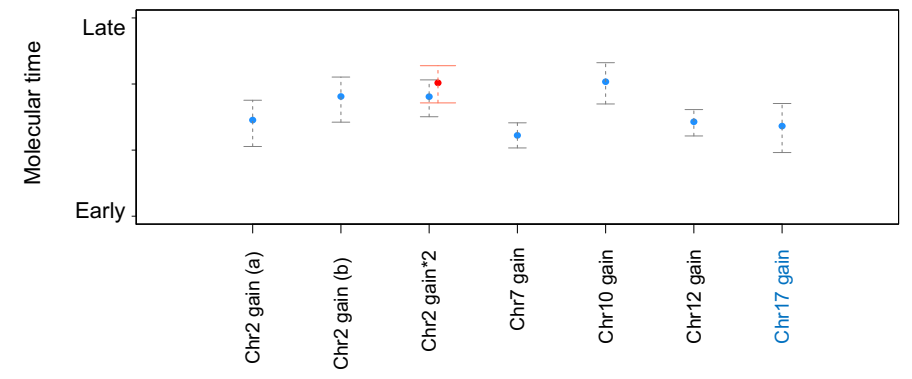

IID_H198432 - Chromosome 10

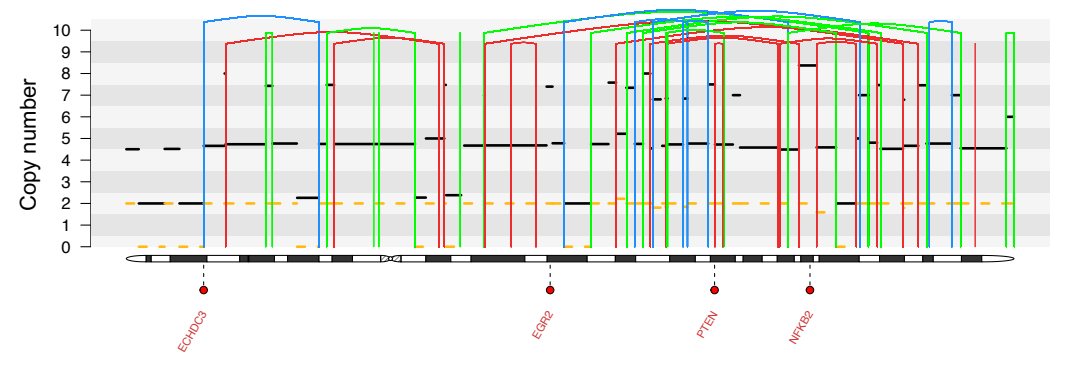

Figure 5 
a

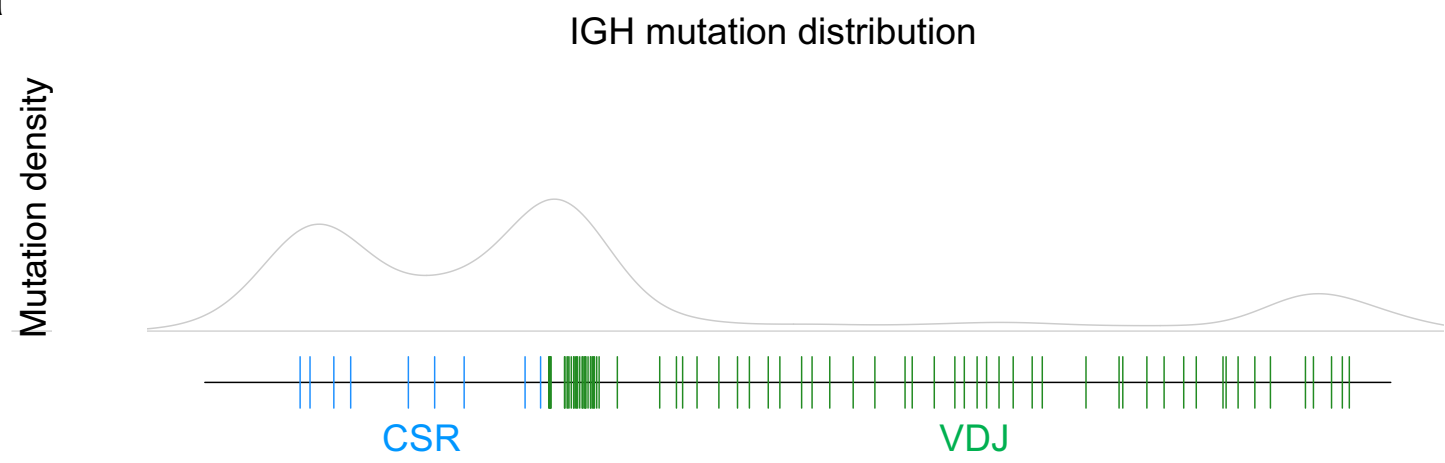

b

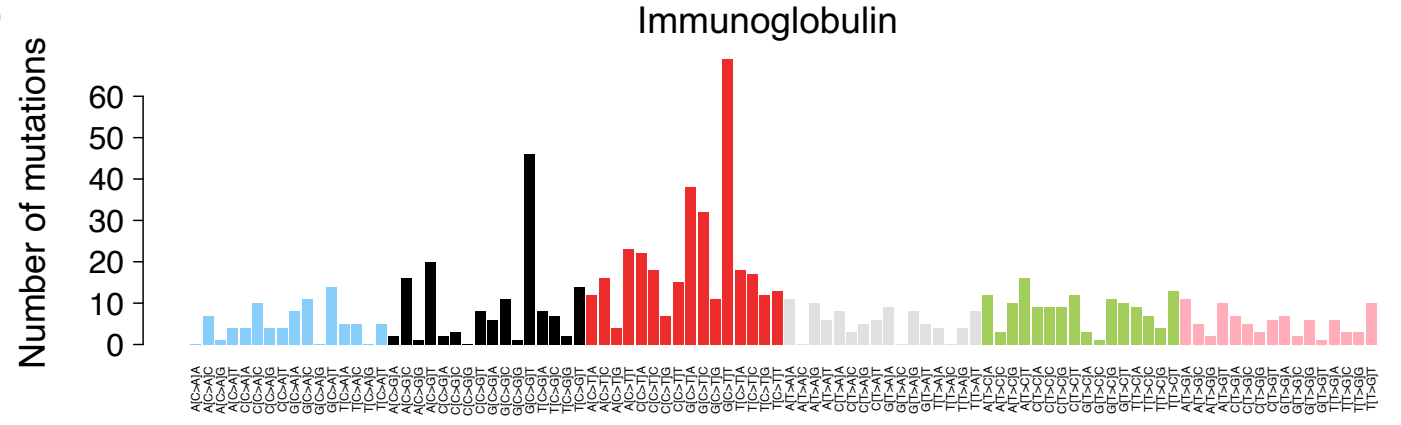

C

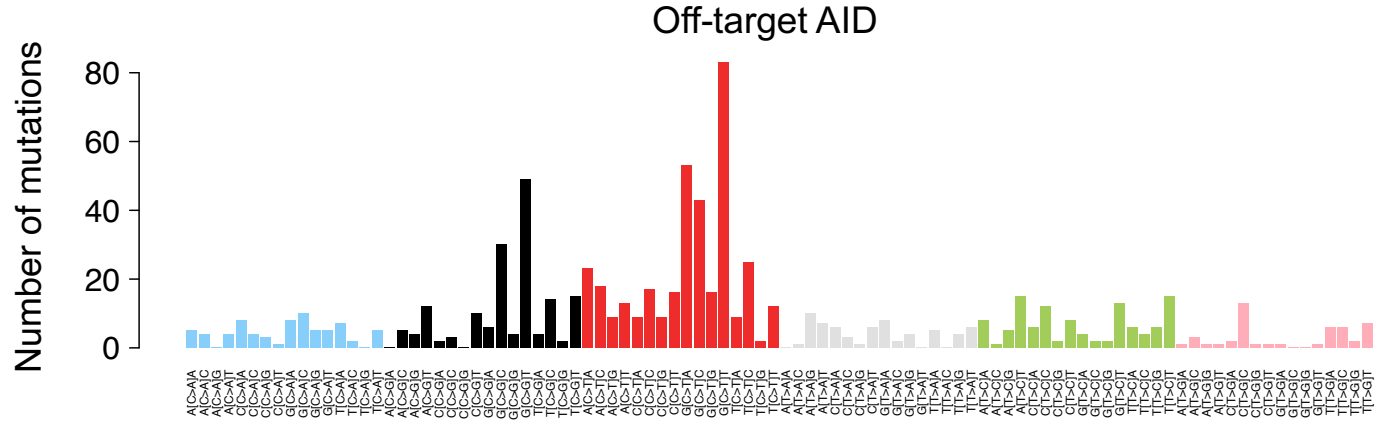

d

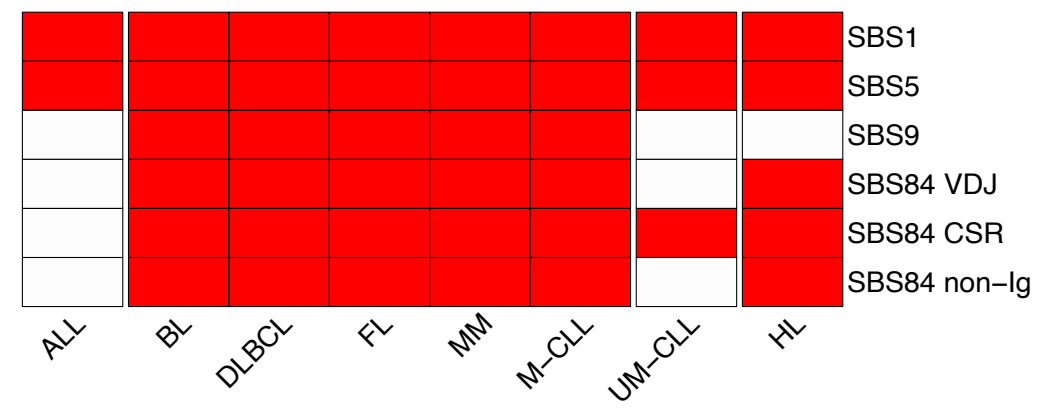

e

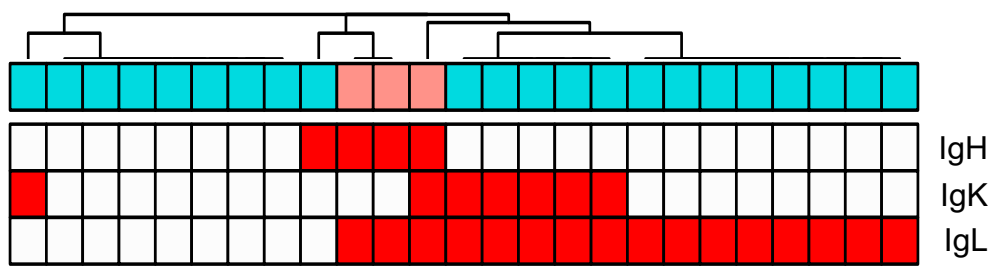

$\square$ V(D)J productive $\square$ V(D)J unproductive

Productive BCR $\square$ Unproductive BCR

f

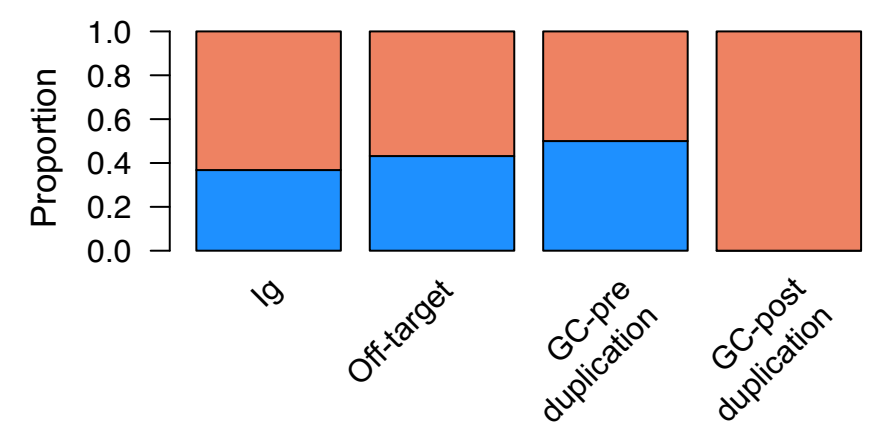

Duplicated

Non-duplicated

Figure 6 


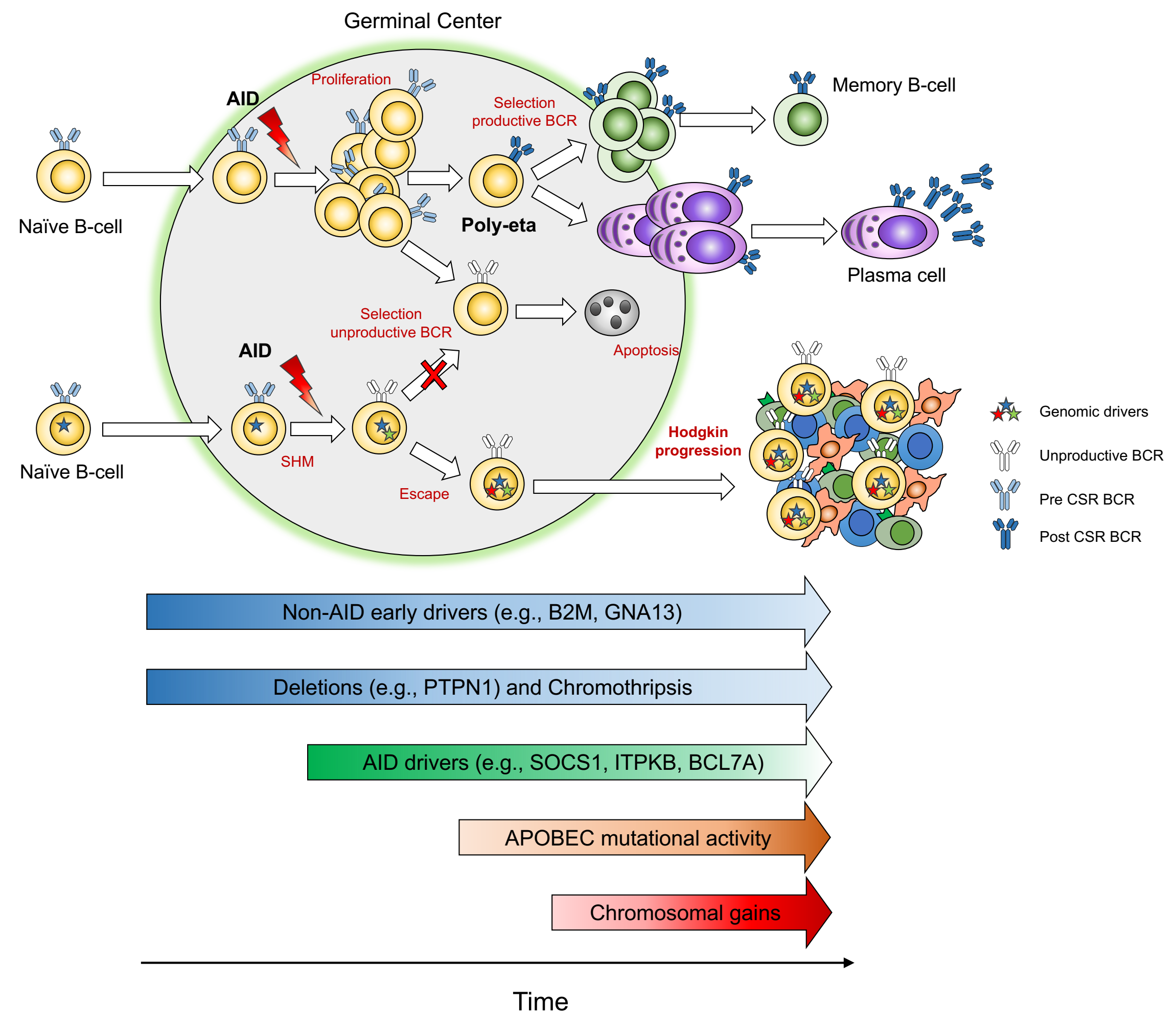

